\title{
Error Analysis of an Implicit Spectral Scheme Applied to the Schrödinger-Benjamin-Ono System
}

\author{
Juan Carlos Muñoz Grajales \\ Departamento de Matemáticas, Universidad del Valle, Calle 13 Nro. 100-00, Cali, Colombia \\ Correspondence should be addressed to Juan Carlos Muñoz Grajales; jcarlmz@yahoo.com
}

Received 30 July 2016; Accepted 10 October 2016

Academic Editor: Wen-Xiu Ma

Copyright ( 2016 Juan Carlos Muñoz Grajales. This is an open access article distributed under the Creative Commons Attribution License, which permits unrestricted use, distribution, and reproduction in any medium, provided the original work is properly cited.

We develop error estimates of the semidiscrete and fully discrete formulations of a Fourier-Galerkin numerical scheme to approximate solutions of a coupled nonlinear Schrödinger-Benjamin-Ono system that describes the motion of two fluids with different densities under capillary-gravity waves in a deep water regime. The accuracy of the numerical solver is checked using some exact travelling wave solutions of the system.

\section{Introduction}

In this paper we shall study numerically the nonlinear onedimensional system (named as the Schrödinger-BenjaminOno system (SBO)):

$$
\begin{gathered}
i \partial_{t} u+\partial_{x}^{2} u=\alpha v u, \\
\partial_{t} v-\gamma \mathscr{H} \partial_{x}^{2} v=\beta\left(|u|^{2}\right)_{x},
\end{gathered}
$$

for $x \in(0, L), t>0$ with periodic spatial boundary conditions. This system describes the motion of two fluids with different densities under capillary-gravity waves in a deep water regime. In this physical phenomenon the long internal wave is described by a wave equation with a dispersive term represented by a nonlocal Hilbert operator, and the short surface wave is described by a Schrödinger type equation. This nonlinear coupled system was derived by Funakoshi and Oikawa [1] in a regime such that the fluid depth of the lower layer is sufficiently large, in comparison with the wavelength of the internal wave. Here $u=u(x, t): \mathbb{R} \times \mathbb{R} \rightarrow \mathbb{C}$ denotes the short wave term and $v=v(x, t): \mathbb{R} \times \mathbb{R} \rightarrow \mathbb{R}$ denotes the long wave term. Furthermore, $\alpha, \beta$ are positive constants, $\gamma \in \mathbb{R}$, and $\mathscr{H}$ denotes the Hilbert transform defined by

$$
\mathscr{H} f(x):=\text { p.v. } \frac{1}{\pi} \int \frac{f(y)}{y-x} d y .
$$

The SBO system also appears in the sonic-Langmuir wave interaction in plasma physics (Karpman [2]), in the capillarygravity interaction waves (Djordjevic and Redekoop [3], Grimshaw [4]), and in the general theory of water-wave interaction in a nonlinear medium (Benney $[5,6]$ ).

An important property of system (1) due to nonlinearity and dispersive effects is that it possesses the so-called travelling wave solutions in the form

$$
\begin{aligned}
& u(x, t)=e^{i w_{p} t} e^{i c(x-c t) / 2} \phi(x-c t), \\
& v(x, t)=\psi(x-c t),
\end{aligned}
$$

with $w_{p}, c \in \mathbb{R}$ and $\phi, \psi$ being periodic real-valued functions or smooth functions such that, for each $n \in \mathbb{N}, \phi^{(n)}(\xi) \rightarrow 0$ and $\psi^{(n)}(\xi) \rightarrow 0$, as $|\xi| \rightarrow \infty$. In this last case, these solutions are called solitary waves. Angulo and Montenegro [7] have proved the existence of even solitary wave solutions 
using the concentration compactness method (Lions $[8,9]$ ) and the theory of symmetric decreasing rearrangements. The existence and stability of a new set of solitary waves were presented in [10] for system (1) and a coupled Schrödinger$\mathrm{KdV}$ model. On the other hand, when $|\gamma| \neq 1$, the nonperiodic initial value problem corresponding to the SBO system has been considered by Bekiranov et al. [11], who proved a well-posedness theory in the Sobolev space $H_{\mathbb{C}}^{s}(\mathbb{R}) \times$ $H_{\mathbb{R}}^{s-1 / 2}(\mathbb{R})$, with $s \geq 0$. When $|\gamma|=1$, Pecher [12] showed the local well-posedness for $s>0$. More recently, Angulo et al. [13] proved the global well-posedness for $s=0$ in the case that $|\gamma| \neq 1$. In the periodic setting, there are only a few results known. For instance, assuming that $|\gamma| \neq$ 0,1 , Angulo et al. [13] showed that system (1) is locally well posed in the Sobolev space $H^{s}(0,2 \pi) \times H^{s-1 / 2}(0,2 \pi)$ for $s \geq 1 / 2$.

In this paper, we shall develop a rigorous analysis of the error of the semidiscrete and fully discrete formulations of a Fourier-Galerkin scheme to approximate solutions of the SBO system (1). The time-stepping method is implemented by using a second-order implicit Crank-Nicholson strategy. The rates of convergence of the semidiscrete and fully discrete schemes are $O\left(N^{s+2-r}\right)$ and $O\left(N^{s+2-r}+\Delta t^{2}\right)$, respectively, where $r>s+2$ depends only on the smoothness of the exact solution, $\Delta t$ is the time step, and $N$ is the number of spatial Fourier modes. The strategy to obtain these rates of convergence follows the one used by Muñoz Grajales [14, 15] and Antonopoulos et al. [16, 17] and Pelloni and Dougalis [18] for other dispersive systems. On the other hand, Rashid and Akram [19] studied the error of an implicit spectral scheme for the SBO system but only in the particular case that $\gamma=0$. Furthermore, Funakoshi and Oikawa [1] computed numerically some approximations to travelling wave solutions of the SBO system. However, an analysis of error of a fully discrete spectral numerical scheme for the complete SBO system has not been performed in previous works to the best of the author's knowledge. This is one motivation for the present study. We point out that system (1) does not have exact solutions in the general case that $\gamma \neq 0$. Therefore, a numerical strategy is very important in order to investigate the properties of the solution space, such as existence of periodic and nonperiodic travelling waves, orbital stability under small initial disturbances, and interactions among these solutions.

The accuracy and convergence rate of the Fourier-spectral scheme proposed in this paper are illustrated by using a family of exact solitary wave solutions of system (1) when $\gamma=0$. In order to apply this scheme in a nonperiodic setting, we approximate the initial value problem for system (1) with $x \in \mathbb{R}$, by the corresponding periodic Cauchy problem for $x \in[0, L]$, with a large spatial period $L$. This type of approximation can be justified by the decay of the solutions of the unrestricted problem as $|x| \rightarrow \infty$.

This paper is organized as follows. In Section 2, we introduce notation and functional spaces which will be used in our work. In Section 3, the analytical properties and convergence of the semidiscrete scheme to approximate solutions of the SBO system are investigated. Section 4 deals with the convergence of the fully discrete scheme that we propose for solving the SBO system. Finally in Section 5, to validate the theoretical results, some numerical experiments using a family of analytical and approximate solutions of the SBO system are performed.

\section{Preliminaries}

We set

$$
\begin{gathered}
L^{2}(0, L):=\left\{f:[0, L] \longrightarrow \mathbb{C},\|f\|_{0}\right. \\
\left.=\left[\int_{0}^{L}|f(x)|^{2} d x\right]^{1 / 2}<\infty\right\},
\end{gathered}
$$

with the inner product

$$
\langle f, g\rangle=\int_{0}^{L} f(x) \overline{g(x)} d x .
$$

The space of all functions of class $C^{k}$ that are $L$-periodic is denoted by $C_{\text {per }}^{k}(0, L), k=0,1,2, \ldots$. Further $C_{\text {per }}=$ $C_{\text {per }}^{0}(0, L)$ is the space of all continuous functions of period

We will denote by $\mathscr{P}$ the space of all infinitely differentiable functions that are $L$-periodic as well as all their derivatives. We say that $T: \mathscr{P} \rightarrow \mathbb{C}$ defines a periodic distribution, that is, $T \in \mathscr{P}^{\prime}$, if $T$ is linear and there exists a sequence $\left(\Psi_{n}\right)_{n \in \mathbb{N}} \subset \mathscr{P}$ such that

$$
\langle T, \varphi\rangle=\lim _{n \rightarrow \infty} \int_{0}^{L} \Psi_{n}(x) \varphi(x) d x, \quad \forall \varphi \in \mathscr{P} .
$$

Let $s \in \mathbb{R}$. The Sobolev space, denoted by $H_{\mathrm{per}}^{s}=H_{\mathrm{per}}^{s}(0, L)$, is defined as

$$
\begin{aligned}
& H_{\text {per }}^{s}(0, L) \\
& \quad=\left\{f \in \mathscr{P}^{\prime}:\|f\|_{s}^{2}=L \sum_{n \in \mathbb{Z}}\left(1+k^{2}\right)^{s}|\widehat{f}(n)|^{2}<\infty\right\},
\end{aligned}
$$

where $\hat{f}: \mathbb{Z} \rightarrow \mathbb{C}$ represents the Fourier transform of $f \in \mathscr{P}^{\prime}$ defined by

$$
\widehat{f}(n)=\frac{1}{L}\left\langle f, e^{-2 \pi i n x / L}\right\rangle, \quad n \in \mathbb{Z} .
$$

In case that $f \in C_{\text {per }}$, we can rewrite $\widehat{f}(n)$ as

$$
\widehat{f}(n)=\frac{1}{L} \int_{0}^{L} f(x) e^{-2 \pi i n x / L} d x, \quad n \in \mathbb{Z} .
$$

It can be shown that, for all $s \in \mathbb{R}, H_{\text {per }}^{s}$ is a Hilbert space with respect to the inner product $\langle\cdot, \cdot\rangle_{s}$ defined as follows:

$$
\langle f, g\rangle_{s}=L \sum_{n \in \mathbb{Z}}\left(1+n^{2}\right)^{s} \hat{f}(n) \overline{\widehat{g}(n)} .
$$

In particular, when $s=0$, we get the Hilbert space denoted by $L_{\text {per }}^{2}=H_{\text {per }}^{0}$. It is important to note that this space is 
isometrically isomorphic to $L^{2}(0, L)$. Further we recall that Parseval's identity holds; that is, for $f \in C_{\text {per }}$

$$
\sum_{n \in \mathbb{Z}}|\widehat{f}(n)|^{2}=\frac{1}{L}\|f\|_{0}^{2}
$$

or, equivalently,

$$
\langle f, g\rangle=L \sum_{n \in \mathbb{Z}} \hat{f}(n) \overline{\widehat{g}}(n)=L\langle\hat{f}, \hat{g}\rangle .
$$

Let $N$ be an even integer and consider the finite dimensional space $S_{N}$ defined by

$$
S_{N}=\operatorname{span}\left\{\frac{1}{\sqrt{L}} e^{2 \pi i n x / L}:-\frac{N}{2} \leq n \leq \frac{N}{2}\right\} .
$$

Remember that the family $\left\{(1 / \sqrt{L}) e^{2 \pi i n x / L}: n \in \mathbb{Z}\right\}$ is an orthonormal and complete system in $L_{\text {per }}^{2}(0, L)$. Let $P_{N}$ be the orthogonal projection $P_{N}: L_{\text {per }}^{2}(0, L) \rightarrow S_{N}$ on the space $S_{N}$ :

$$
P_{N} g:=\sum_{n=-N / 2}^{N / 2} \widehat{g}_{n} \phi_{n}
$$

with

$$
\begin{aligned}
\phi_{n}(x) & =e^{2 \pi i n x / L}, \\
\widehat{g}_{n} & =\frac{1}{L} \int_{0}^{L} g(x) \bar{\phi}_{n}(x) d x .
\end{aligned}
$$

This operator has the following properties (see [20, 21]): For any $g \in L_{\text {per }}^{2}(0, L)$,

$$
\left\langle P_{N} g-g, \phi\right\rangle=0, \quad \forall \phi \in S_{N} .
$$

Furthermore, given integers $0 \leq s \leq \alpha$, there exists a constant $C$ independent of $N$ such that, for any $g \in H_{\text {per }}^{\alpha}(0, L)$,

$$
\left\|P_{N} g-g\right\|_{s} \leq C N^{s-\alpha}\|g\|_{\alpha} .
$$

In what follows, for a positive integer $k, C^{k}([0, T], X)$ denotes the space of $k$-times continuously differentiable maps from $[0, T]$ onto a Banach space $X$.

\section{The Semidiscrete Scheme}

Let us consider the SBO system

$$
\begin{aligned}
i \partial_{t} u+\partial_{x}^{2} u-\alpha u v & =0, \quad x \in(0, L), t>0, \\
\partial_{t} v-\gamma \mathscr{H}\left(\partial_{x}^{2} v\right)-\beta \partial_{x}\left(|u|^{2}\right) & =0,
\end{aligned}
$$

subject to the initial conditions $u(x, 0)=u_{0}(x), v(x, 0)=$ $v_{0}(x)$ and $u, v L$-periodic complex valued functions in the variable $x$.

The semidiscrete Fourier-Galerkin spectral scheme to solve problem (18) is to find $u_{N}, v_{N} \in C\left([0, T], S_{N}\right)$ such that

$$
\begin{aligned}
\left\langle i \partial_{t} u_{N}+\partial_{x}^{2} u_{N}-\alpha u_{N} v_{N}, \phi\right\rangle & =0, \\
\left\langle\partial_{t} v_{N}-\gamma \mathscr{H} \partial_{x}^{2} v_{N}-\beta \partial_{x}\left(\left|u_{N}\right|^{2}\right), \psi\right\rangle & =0, \\
u_{N}(0) & =P_{N}\left(u_{0}\right), \\
v_{N}(0) & =P_{N}\left(v_{0}\right),
\end{aligned}
$$

for any $\phi, \psi \in S_{N}$ and $0 \leq t \leq T$.
Theorem 1. Let $s \geq 2$ be an integer, and let $u, v \in C^{1}([0$, $\left.T], H_{\text {per }}^{r}(0, L)\right)$ be the classical solution of problem (18) for some integer $r>s+2$. Let $u_{N}, v_{N} \in C^{1}\left(\left[0, T_{N}\right], S_{N}\right)$ be the solution of the semidiscrete formulation (19) defined until some maximal time $0<T_{N}<T$. Then, for $N$ sufficiently large, $u_{N}, v_{N}$ can be extended to the whole interval $[0, T]$ and there exists a constant $C>0$, independent of $N$, $t$, such that

$$
\left\|u(t)-u_{N}(t)\right\|_{s+1}+\left\|v(t)-v_{N}(t)\right\|_{s} \leq C N^{s+2-r},
$$

for any $0 \leq t \leq T$.

Proof. Let

$$
\begin{aligned}
& e_{1}=u-u_{N}=\left(u-P_{N} u\right)+\left(P_{N} u-u_{N}\right):=\xi_{1}+\eta_{1}, \\
& e_{2}=v-v_{N}=\left(v-P_{N} v\right)+\left(P_{N} v-v_{N}\right):=\xi_{2}+\eta_{2} .
\end{aligned}
$$

Observe that by the fact that $P_{N}$ is an orthogonal projection and if $s \in \mathbb{Z}_{+}$then $\partial_{x}^{s} \phi \in S_{N}$ for any $\phi \in S_{N}$, and thus

$$
\left\langle\xi_{l}, \phi\right\rangle_{s}=0, \quad \phi \in S_{N}, l=1,2 ;
$$

that is,

$$
\begin{gathered}
\left\langle u-P_{N} u, \phi\right\rangle_{s}=0, \\
\left\langle v-P_{N} v, \phi\right\rangle_{s}=0,
\end{gathered}
$$

for any $\phi \in S_{N}$. Therefore, by virtue of (17)

$$
\left\|e_{l}\right\|_{s} \leq\left\|\xi_{l}\right\|_{s}+\left\|\eta_{l}\right\|_{s} \leq C N^{s-r}+\left\|\eta_{l}\right\|_{s}, \quad l=1,2 .
$$

Now, by combining the equations satisfied by the pairs $(u, v)$, $\left(u_{N}, v_{N}\right)$, we arrive at

$$
\begin{aligned}
& \left\langle i \partial_{t}\left(u-u_{N}\right)+\partial_{x}^{2}\left(u-u_{N}\right), \phi\right\rangle_{s} \\
& =\alpha\left\langle v u-v_{N} u_{N}, \phi\right\rangle_{s}, \\
& \left\langle\partial_{t}\left(v-v_{N}\right)_{t}-\gamma \mathscr{H} \partial_{x}^{2}\left(v-v_{N}\right), \psi\right\rangle_{s} \\
& =\beta\left\langle\partial_{x}\left(|u|^{2}-\left|u_{N}\right|^{2}\right), \psi\right\rangle_{s} .
\end{aligned}
$$

As a consequence,

$$
\begin{gathered}
\left\langle i \partial_{t} e_{1}+\partial_{x}^{2} e_{1}, \phi\right\rangle_{s}=\alpha\left\langle v u-v_{N} u_{N}, \phi\right\rangle_{s}, \\
\left\langle\partial_{t} e_{2}-\gamma \mathscr{H} \partial_{x}^{2} e_{2}, \psi\right\rangle_{s}=\beta\left\langle\partial_{x}\left(|u|^{2}-\left|u_{N}\right|^{2}\right), \psi\right\rangle_{s} .
\end{gathered}
$$

Letting $\phi=\eta_{1}$ in (26), we obtain

$$
i\left\langle\partial_{t} e_{1}, \eta_{1}\right\rangle_{s}+\left\langle\partial_{x}^{2} e_{1}, \eta_{1}\right\rangle_{s}=\alpha\left\langle v u-v_{N} u_{N}, \eta_{1}\right\rangle_{s} .
$$

Since $e_{1}=\xi_{1}+\eta_{1}$,

$$
\begin{aligned}
& i\left\langle\partial_{t} \xi_{1}+\partial_{t} \eta_{1}, \eta_{1}\right\rangle_{s}+\left\langle\partial_{x}^{2} \xi_{1}+\partial_{x}^{2} \eta_{1}, \eta_{1}\right\rangle_{s} \\
& \quad=\alpha\left\langle v u-v_{N} u_{N}, \eta_{1}\right\rangle_{s} .
\end{aligned}
$$

Note that due to $\partial_{t} \eta_{1} \in S_{N}$ thus $\left\langle\xi_{1}, \partial_{t} \eta_{1}\right\rangle_{s}=0$ and

$$
\partial_{t}\left\langle\xi_{1}, \eta_{1}\right\rangle_{s}=\left\langle\partial_{t} \xi_{1}, \eta_{1}\right\rangle_{s}+\left\langle\xi_{1}, \partial_{t} \eta_{1}\right\rangle_{s}=0 .
$$


As a consequence, $\left\langle\partial_{t} \xi_{1}, \eta_{1}\right\rangle_{s}=0$. Furthermore, since $\partial_{x}^{2} \eta_{1} \epsilon$ $S_{N}$ and using integration by parts, we have

$$
\left\langle\partial_{x}^{2} \xi_{1}, \eta_{1}\right\rangle_{s}=\left\langle\xi_{1}, \partial_{x}^{2} \eta_{1}\right\rangle_{s}=0 .
$$

Therefore,

$$
\begin{aligned}
i\left\langle\partial_{t} \eta_{1}, \eta_{1}\right\rangle_{s}-\left\|\partial_{x} \eta_{1}\right\|^{2} & =\alpha\left\langle v u-v_{N} u_{N}, \eta_{1}\right\rangle_{s}, \\
\frac{i}{2} \partial_{t}\left\|\eta_{1}\right\|_{s}^{2}-\left\|\partial_{x} \eta_{1}\right\|_{s}^{2} & =\alpha\left\langle v u-v_{N} u_{N}, \eta_{1}\right\rangle_{s} .
\end{aligned}
$$

Now taking imaginary part

$$
\frac{1}{2} \partial_{t}\left\|\eta_{1}\right\|_{s}^{2}=\alpha \operatorname{Im}\left\langle v u-v_{N} u_{N}, \eta_{1}\right\rangle_{s} .
$$

To bound the nonlinear terms, let us observe that

$$
\begin{aligned}
& \left|\alpha \operatorname{Im}\left\langle v u-v_{N} u_{N}, \eta_{1}\right\rangle_{s}\right| \leq|\alpha|\left|\left\langle v u-v_{N} u_{N}, \eta_{1}\right\rangle_{s}\right| \\
& \quad \leq|\alpha|\left\|v u-v_{N} u_{N}\right\|_{s}\left\|\eta_{1}\right\|_{s} \\
& \quad \leq C\left(\left\|v u-v_{N} u_{N}\right\|_{s}^{2}+\left\|\eta_{1}\right\|_{s}^{2}\right),
\end{aligned}
$$

and as the space $H_{\mathrm{per}}^{s}$ is an algebra for $s>1 / 2$,

$$
\begin{aligned}
\left\|v u-v_{N} u_{N}\right\|_{s} & =\left\|u\left(v-v_{N}\right)+v_{N}\left(u-u_{N}\right)\right\|_{s} \\
& \leq\|u\|_{s}\left\|v-v_{N}\right\|_{s}+\left\|v_{N}\right\|_{s}\left\|u-u_{N}\right\|_{s} \\
& \leq\|u\|_{s}\left\|e_{2}\right\|_{s}+\left\|v_{N}\right\|_{s}\left\|e_{1}\right\|_{s} .
\end{aligned}
$$

From hypothesis, $u, v \in C\left([0, T], H_{\text {per }}^{r}(0, L)\right)$, with $r>s+$ 2 , and thus there exists a positive constant $M>0$ such that

$$
\max _{t \in[0, T]}\left(\|u(t)\|_{s}+\|v(t)\|_{s}\right)<M .
$$

Further suppose that $0<T_{N}<T$ is the largest value such that

$$
\max _{t \in\left[0, T_{N}\right]}\left(\left\|u_{N}(t)\right\|_{s}+\left\|v_{N}(t)\right\|_{s}\right)<2 M .
$$

Because of the embedding to $H_{\text {per }}^{s} \subset L^{\infty}$, for $s>1 / 2$, we obtain from (33) and inequality (34)

$$
\frac{1}{2} \partial_{t}\left\|\eta_{1}\right\|_{s}^{2} \leq C\left(N^{2(s-r)}+\left\|\eta_{1}\right\|_{s}^{2}+\left\|\eta_{2}\right\|_{s}^{2}\right) .
$$

On the other hand, from (27),

$$
\begin{aligned}
\left\langle\partial_{t} \xi_{2}+\partial_{t} \eta_{2}, \psi\right\rangle_{s}= & \beta\left\langle\partial_{x}\left(|u|^{2}-\left|u_{N}\right|^{2}\right), \psi\right\rangle_{s} \\
& +\gamma\left\langle\mathscr{H} \partial_{x}^{2} \xi_{2}, \psi\right\rangle_{s} \\
& +\gamma\left\langle\mathscr{H} \partial_{x}^{2} \eta_{2}, \psi\right\rangle_{s} .
\end{aligned}
$$

Letting $\psi=\eta_{2}$,

$$
\begin{aligned}
& \left\langle\partial_{t} \xi_{2}, \eta_{2}\right\rangle_{s}+\left\langle\partial_{t} \eta_{2}, \eta_{2}\right\rangle_{s} \\
& =\beta\left\langle\partial_{x}\left(|u|^{2}-\left|u_{N}\right|^{2}\right), \eta_{2}\right\rangle_{s}+\gamma\left\langle\mathscr{H} \partial_{x}^{2} \xi_{2}, \eta_{2}\right\rangle_{s} \\
& \quad+\gamma\left\langle\mathscr{H} \partial_{x}^{2} \eta_{2}, \eta_{2}\right\rangle_{s} .
\end{aligned}
$$

Then, following an analogous procedure as above, we can obtain that

$$
\left\langle\partial_{t} \xi_{2}, \eta_{2}\right\rangle_{s}=0
$$

and, taking into account the property of the Hilbert transform operator

$$
\left\langle\mathscr{H} \partial_{x}^{2} \eta_{2}, \eta_{2}\right\rangle_{s}=0
$$

we get that

$$
\begin{aligned}
\frac{1}{2} \partial_{t}\left\|\eta_{2}\right\|_{s}^{2}= & \beta\left\langle\partial_{x}\left(|u|^{2}-\left|u_{N}\right|^{2}\right), \eta_{2}\right\rangle_{s} \\
& +\gamma\left\langle\mathscr{H} \partial_{x}^{2} \xi_{2}, \eta_{2}\right\rangle_{s} .
\end{aligned}
$$

Again, to bound the nonlinear terms, let us note that

$$
\begin{aligned}
& \left|\beta\left\langle\partial_{x}\left(|u|^{2}-\left|u_{N}\right|^{2}\right), \eta_{2}\right\rangle_{s}\right| \\
& \quad \leq C\left(\left\|\partial_{x}\left(|u|^{2}-\left|u_{N}\right|^{2}\right)\right\|_{s}^{2}+\left\|\eta_{2}\right\|_{s}^{2}\right) .
\end{aligned}
$$

But

$$
\begin{aligned}
& \left\|\partial_{x}\left(|u|^{2}-\left|u_{N}\right|^{2}\right)\right\|_{s}=\left\|\partial_{x}(u \bar{u})-\partial_{x}\left(u_{N} \overline{u_{N}}\right)\right\|_{s} \\
& \quad \leq C\left(\|\bar{u}\|_{s}\left\|\partial_{x}\left(u-u_{N}\right)\right\|_{s}+\left\|\partial_{x} u_{N}\right\|_{s}\left\|\bar{u}-\overline{u_{N}}\right\|_{s}\right. \\
& \left.\quad+\left\|\partial_{x} \bar{u}\right\|_{s}\left\|u-u_{N}\right\|_{s}+\left\|u_{N}\right\|_{s}\left\|\partial_{x}\left(\bar{u}-\overline{u_{N}}\right)\right\|_{s}\right) \\
& \quad \leq C\left(\left\|\partial_{x} \xi_{1}\right\|_{s}+\left\|\partial_{x} \eta_{1}\right\|_{s}+\left\|\xi_{1}\right\|+\left\|\eta_{1}\right\|_{s}\right) \\
& \quad \leq C\left(N^{s+1-r}+\left\|\eta_{1}\right\|_{s}+\left\|\partial_{x} \eta_{1}\right\|_{s}\right) .
\end{aligned}
$$

Therefore, from (43) and $\left\|\mathscr{H} \partial_{x}^{2} \xi_{2}\right\|_{s} \leq\left\|\xi_{2}\right\|_{s+2} \leq N^{s+2-r}$, we arrive at

$$
\begin{aligned}
& \frac{1}{2} \partial_{t}\left\|\eta_{2}\right\|_{s}^{2} \leq C\left(\left\|\partial_{x}\left(|u|^{2}-\left|u_{N}\right|^{2}\right)\right\|_{s}^{2}+\left\|\eta_{2}\right\|_{s}^{2}\right. \\
& \left.+\left\|\mathscr{H} \partial_{x}^{2} \xi_{2}\right\|_{s}^{2}+\left\|\eta_{2}\right\|_{s}^{2}\right) \leq C\left(N^{2(s+1-r)}+\left\|\eta_{1}\right\|_{s}^{2}\right. \\
& \left.+\left\|\partial_{x} \eta_{1}\right\|_{s}^{2}+\left\|\eta_{2}\right\|_{s}^{2}+\left\|\xi_{2}\right\|_{s}^{2}\right) \leq C\left(N^{2(s+1-r)}+\left\|\eta_{1}\right\|_{s}^{2}\right. \\
& \left.+\left\|\eta_{2}\right\|_{s}^{2}+N^{2(s+2-r)}+\left\|\partial_{x} \eta_{1}\right\|_{s}^{2}\right) \leq C\left(\left\|\eta_{1}\right\|_{s}^{2}+\left\|\eta_{2}\right\|_{s}^{2}\right. \\
& \left.\quad+N^{2(s+2-r)}+\left\|\partial_{x} \eta_{1}\right\|_{s}^{2}\right) .
\end{aligned}
$$

Letting $\phi=\partial_{t} \eta_{1}$ in (26), we have that

$$
\begin{aligned}
& i\left\langle\partial_{t} e_{1}, \partial_{t} \eta_{1}\right\rangle_{s}+\left\langle\partial_{x}^{2} e_{1}, \partial_{t} \eta_{1}\right\rangle_{s}-\alpha\left\langle v u-v_{N} u_{N}, \partial_{t} \eta_{1}\right\rangle_{s} \\
& \quad=0 .
\end{aligned}
$$

Then we can replace $e_{1}=\xi_{1}+\eta_{1}$ in the previous equation to get

$$
\begin{aligned}
& i\left\langle\partial_{t} \xi_{1}, \partial_{t} \eta_{1}\right\rangle_{s}+i\left\langle\partial_{t} \eta_{1}, \partial_{t} \eta_{1}\right\rangle_{s}+\left\langle\partial_{x}^{2} \xi_{1}, \partial_{t} \eta_{1}\right\rangle_{s} \\
& \quad+\left\langle\partial_{x}^{2} \eta_{1}, \partial_{t} \eta_{1}\right\rangle_{s}-\alpha\left\langle v u-v_{N} u_{N}, \partial_{t} \eta_{1}\right\rangle_{s}=0
\end{aligned}
$$


Therefore, since $\partial_{t} \eta_{1} \in S_{N}$ and $i\left\langle\partial_{t} \xi_{1}, \partial_{t} \eta_{1}\right\rangle_{s}=0$ and using integration by parts, it follows that

$$
\begin{aligned}
& \left\langle\partial_{x}^{2} \xi_{1}, \partial_{t} \eta_{1}\right\rangle_{s}=\left\langle\xi_{1}, \partial_{x}^{2} \partial_{t} \eta_{1}\right\rangle_{s}=0, \\
& \left\langle\partial_{x}^{2} \eta_{1}, \partial_{t} \eta_{1}\right\rangle_{s}=-\left\langle\partial_{x} \eta_{1}, \partial_{x} \partial_{t} \eta_{1}\right\rangle_{s} .
\end{aligned}
$$

As a consequence, (48) implies that

$$
i\left\|\partial_{t} \eta_{1}\right\|_{s}^{2}-\frac{1}{2} \partial_{t}\left\|\partial_{x} \eta_{1}\right\|_{s}^{2}-\alpha\left\langle v u-v_{N} u_{N}, \partial_{t} \eta_{1}\right\rangle_{s}=0
$$

Taking imaginary part of the previous equation and using Cauchy-Schwartz and Hölder inequalities,

$$
\begin{aligned}
\left\|\partial_{t} \eta_{1}\right\|_{s}^{2} & =\alpha \operatorname{Im}\left(\left\langle v u-u_{N} v_{N}, \partial_{t} \eta_{1}\right\rangle_{s}\right) \\
& \leq\left\|v u-v_{N} u_{N}\right\|_{s}\left\|\partial_{t} \eta_{1}\right\|_{s} \\
& \leq \alpha\left(\frac{1}{4 \epsilon}\left\|v u-v_{N} u_{N}\right\|_{s}^{2}+\epsilon\left\|\partial_{t} \eta_{1}\right\|_{s}^{2}\right),
\end{aligned}
$$

where $\epsilon>0$. The nonlinear terms in the right-hand side of the last equation can be estimated as in (34), and then, choosing $\epsilon>0$ small enough, we obtain the estimate

$$
\left\|\partial_{t} \eta_{1}\right\|_{s}^{2} \leq C\left(N^{2(s-r)}+\left\|\eta_{1}\right\|_{s}^{2}+\left\|\eta_{2}\right\|_{s}^{2}\right)
$$

On the other hand, we can take real part of (50) to achieve

$$
\begin{aligned}
\frac{1}{2} \partial_{t}\left\|\partial_{x} \eta_{1}\right\|_{s}^{2} & =-\alpha\left\langle v u-v_{N} u_{N}, \partial_{t} \eta_{1}\right\rangle_{s} \\
& \leq C\left\|v u-v_{N} u_{N}\right\|_{s}\left\|\partial_{t} \eta_{1}\right\|_{s} \\
& \leq C\left(\left\|v u-v_{N} u_{N}\right\|_{s}^{2}+\left\|\partial_{t} \eta_{1}\right\|_{s}^{2}\right) \\
& \leq C\left(N^{2(s-r)}+\left\|\eta_{1}\right\|_{s}^{2}+\left\|\eta_{2}\right\|_{s}^{2}+\left\|\partial_{t} \eta_{1}\right\|_{s}^{2}\right)
\end{aligned}
$$

Taking into account the previous results, we arrive at

$$
\begin{gathered}
\partial_{t}\left(\left\|\eta_{1}\right\|_{s}^{2}+\left\|\partial_{x} \eta_{1}\right\|_{s}^{2}+\left\|\eta_{2}\right\|_{s}^{2}\right) \leq C\left(N^{2(s-r)}+\left\|\eta_{1}\right\|_{s}^{2}\right. \\
\left.+\left\|\eta_{2}\right\|_{s}^{2}+\left\|\partial_{t} \eta_{1}\right\|_{s}^{2}+N^{2(s+2-r)}+\left\|\partial_{x} \eta_{1}\right\|_{s}^{2}\right) \\
\leq C\left(N^{2(s+2-r)}+\left\|\eta_{1}\right\|_{s}^{2}+\left\|\eta_{2}\right\|_{s}^{2}+\left\|\partial_{x} \eta_{1}\right\|_{s}^{2}\right) .
\end{gathered}
$$

Thus, using Gronwall's lemma,

$$
\left\|\eta_{1}\right\|_{s+1}+\left\|\eta_{2}\right\|_{s} \leq C N^{s+2-r}
$$

for $0 \leq t \leq T_{N}$. Observe that, for any $0 \leq t \leq T_{N}$,

$$
\begin{aligned}
\left\|u_{N}(t)\right\|_{s}+\left\|v_{N}(t)\right\|_{s}= & \left\|u_{N}-P_{N} u+P_{N} u-u+u\right\|_{s} \\
& +\left\|v_{N}-P_{N} v+P_{N} v-v+v\right\|_{s} \\
\leq & \left\|u_{N}-P_{N} u\right\|_{s}+\left\|P_{N} u-u\right\|_{s} \\
& +\|u\|_{s}+\left\|v_{N}-P_{N} v\right\|_{s} \\
& +\left\|P_{N} v-v\right\|_{s}+\|v\|_{s} \\
\leq & \left\|\eta_{1}\right\|_{s}+\left\|\xi_{1}\right\|_{s}+\|u\|_{s}+\left\|\eta_{2}\right\|_{s} \\
& +\left\|\xi_{2}\right\|_{s}+\|v\|_{s} \\
\leq & C\left(N^{s+2-r}+N^{s-r}\right)+M \\
< & 2 M,
\end{aligned}
$$

for $N$ large enough and $r>s+2$. This fact contradicts the maximality of $T_{N}$. Thus the solutions $u_{N}, v_{N}$ of the semidiscrete formulation can be extended for any $0 \leq t \leq T$, and inequality (55) is satisfied for any $t \in[0, T]$. Finally, from (24) the result follows.

\section{The Fully Discrete Scheme}

The fully discrete Crank-Nicholson scheme to discretize system (18) consists in finding a sequence $\left\{u_{N}^{n}, v_{N}^{n}\right\}$ of elements of $S_{N} \times S_{N}$, such that, for all $n=1,2, \ldots, M-1$, we have

$$
\begin{aligned}
& \left\langle i\left(\frac{u_{N}^{n+1}-u_{N}^{n}}{\Delta t}\right)+\partial_{x}^{2}\left(\frac{u_{N}^{n+1}+u_{N}^{n}}{2}\right)\right. \\
& \left.-\alpha\left(\frac{u_{N}^{n+1}+u_{N}^{n}}{2}\right)\left(\frac{v_{N}^{n+1}+v_{N}^{n}}{2}\right), \phi\right\rangle=0, \\
& \left\langle\frac{v_{N}^{n+1}-v_{N}^{n}}{\Delta t}-\gamma \mathscr{H} \partial_{x}^{2}\left(\frac{v_{N}^{n+1}+v_{N}^{n}}{2}\right)\right. \\
& \left.-\beta \partial_{x}\left(\left|\frac{u_{N}^{n+1}+u_{N}^{n}}{2}\right|^{2}\right), \psi\right\rangle=0,
\end{aligned}
$$

for all $\phi, \psi \in S_{N}$ and subject to $u_{N}^{0}=P_{N}\left(u_{0}\right), v_{N}^{0}=P_{N}\left(v_{0}\right)$. Here $\Delta t$ is a time step chosen together with a positive integer $M$ such that $M \Delta t=T$. Furthermore we define $t_{n}=n \Delta t$, $n=0,1,2, \ldots, M$.

Theorem 2. Let $s \geq 2$ be an integer, and let $(u, v) \in C^{3}([0$, $\left.T], H_{\text {per }}^{r}(0, L)\right)$ be a classical solution of system (18) with $r>$ $s+2$ integer. Let $\left(u_{N}, v_{N}\right) \in C^{1}\left([0, T], S_{N}\right)$ be the solution of the semidiscrete formulation (19) and let $\left\{\left(u_{N}^{n}, v_{N}^{n}\right)\right\}$ be the solution of the fully discrete scheme (57) such that $\left\|u_{N}^{n}\right\|_{s}+\left\|v_{N}^{n}\right\|_{s} \leq B$, for some sufficiently large constant $B>0$, independent of $n, N$, and $\Delta t$. If $u_{N}^{0}=u_{N}(0), v_{N}^{0}=v_{N}(0)$, then, with the assumption that $u_{0}, v_{0} \in H_{\text {per }}^{r}(0, L)$, there exists a constant $C$ independent 
of $N$ and $\Delta t$ such that if $N$ is large enough and $\Delta t$ sufficiently small, we have that

$$
\begin{aligned}
& \max _{0 \leq n \leq M}\left\{\left\|u\left(t_{n}\right)-u_{N}^{n}\right\|_{s+1}+\left\|v\left(t_{n}\right)-v_{N}^{n}\right\|_{s}\right\} \\
& \quad \leq C\left(N^{s+2-r}+\Delta t^{2}\right) .
\end{aligned}
$$

Proof. For a function $f$, let us introduce the following notation:

$$
\begin{aligned}
& f_{t}^{n}=\frac{1}{\Delta t}\left(f^{n+1}-f^{n}\right), \\
& \hat{f}^{n}=\frac{1}{2}\left(f^{n+1}+f^{n}\right),
\end{aligned}
$$

where $f^{n}:=f\left(t_{n}\right)$. Thus the fully discrete formulation (57) can be rewritten as

$$
\begin{aligned}
\left\langle i u_{t}^{n}+\partial_{x}^{2} \widehat{u}_{N}^{n}-\alpha \widehat{u}_{N}^{n} \widehat{v}_{N}^{n}, \phi\right\rangle_{s} & =0, \\
\left\langle v_{t}^{n}-\gamma \mathscr{H} \partial_{x}^{2}\left(\widehat{v}_{N}^{n}\right)-\beta \partial_{x}\left|\widehat{u}_{N}^{n}\right|^{2}, \psi\right\rangle_{s} & =0, \\
u_{N}^{0} & =P_{N}\left(u_{0}\right), \\
v_{N}^{0} & =P_{N}\left(v_{0}\right) .
\end{aligned}
$$

Observe that

$$
\left\langle u_{t}^{n}, 2 \widehat{u}^{n}\right\rangle_{s}=\frac{1}{\Delta t}\left(\left\|u^{n+1}\right\|_{s}^{2}-\left\|u^{n}\right\|_{s}^{2}\right) .
$$

We also introduce the notation

$$
\begin{aligned}
e_{1}^{n} & =u^{n}-u_{N}^{n}=\left(u^{n}-P_{N} u^{n}\right)+\left(P_{N} u^{n}-u_{N}^{n}\right) \\
& :=\xi_{1}^{n}+\eta_{1}^{n}, \\
e_{2}^{n} & =v^{n}-v_{N}^{n}=\left(v^{n}-P_{N} v^{n}\right)+\left(P_{N} v^{n}-v_{N}^{n}\right) \\
& :=\xi_{2}^{n}+\eta_{2}^{n},
\end{aligned}
$$

where $u^{n}:=u\left(t_{n}\right), v^{n}:=v\left(t_{n}\right)$. Observe that $\left\langle\xi_{l}^{n}, \phi\right\rangle_{s}=0$, $l=1,2$, for any $\phi \in S_{N}$, and, furthermore,

$$
\left\|e_{l}^{n}\right\|_{s} \leq\left\|\xi_{l}^{n}\right\|_{s}+\left\|\eta_{l}^{n}\right\|_{s} \leq C N^{s-r}+\left\|\eta_{l}^{n}\right\|_{s}, \quad l=1,2 .
$$

Combining the equations satisfied by $(u, v),\left(u_{N}, v_{N}\right)$, one can get

$$
\begin{aligned}
& \left\langle i e_{1 t}^{n}, \phi\right\rangle_{s}+\left\langle\partial_{x}^{2} \widehat{e}_{1}^{n}, \phi\right\rangle_{s} \\
& -\alpha\left\langle\widehat{u}^{n} \widehat{v}^{n}-\widehat{u}_{N}^{n} \widehat{v}_{N}^{n}, \phi\right\rangle_{s}=\left\langle\tau_{1}^{n}, \phi\right\rangle_{s}, \\
& \left\langle e_{2 t}^{n}, \psi\right\rangle_{s}-\gamma\left\langle\mathscr{H} \partial_{x}^{2} \widehat{e}_{2}^{n}, \psi\right\rangle_{s} \\
& -\beta\left\langle\partial_{x}\left(\left|\hat{u}^{n}\right|^{2}-\left|\widehat{u}_{N}^{n}\right|^{2}\right), \psi\right\rangle_{s}=\left\langle\tau_{2}^{n}, \psi\right\rangle_{s},
\end{aligned}
$$

where

$$
\begin{aligned}
\tau_{1}^{n}:= & i u_{t}^{n}-i \partial_{t} u^{n}+\partial_{x}^{2} \widehat{u}^{n}-\partial_{x}^{2} u^{n}+\alpha\left(u^{n} v^{n}-\widehat{u}^{n} \widehat{v}^{n}\right), \\
\tau_{2}^{n}:= & v_{t}^{n}-\partial_{t} v^{n}-\gamma \mathscr{H} \partial_{x}^{2} \widehat{v}^{n}+\gamma \mathscr{H} \partial_{x}^{2} v^{n} \\
& +\beta \partial_{x}\left(\left|u^{n}\right|^{2}-\left|\widehat{u}^{n}\right|^{2}\right) .
\end{aligned}
$$

In order to estimate the quantities $\tau_{1}^{n}, \tau_{2}^{n}$, let us rewrite them as

$$
\begin{aligned}
\tau_{1}^{n}= & i\left(\frac{u^{n+1}-u^{n}}{\Delta t}\right)-i \partial_{t} u+\partial_{x}^{2}\left(\frac{u^{n+1}+u^{n}}{2}\right)-\partial_{x}^{2} u^{n} \\
& +\alpha u^{n} v^{n}-\alpha\left(\frac{u^{n+1}+u^{n}}{2}\right)\left(\frac{v^{n+1}+v^{n}}{2}\right), \\
\tau_{2}^{n}= & \frac{v^{n+1}-v^{n}}{\Delta t}-\partial_{t} v^{n}-\gamma \mathscr{H} \partial_{x}^{2}\left(\frac{v^{n+1}+v^{n}}{2}\right) \\
& +\gamma \mathscr{H} \partial_{x}^{2} v^{n}+\beta \partial_{x}\left(\left|u^{n}\right|^{2}-\left|\frac{u^{n+1}+u^{n}}{2}\right|^{2}\right) .
\end{aligned}
$$

Therefore

$$
\begin{aligned}
\tau_{1}^{n} & =i\left(\frac{u^{n}+\Delta t \partial_{t} u^{n}+(1 / 2) \Delta t^{2} \partial_{t}^{2} u^{n}-u^{n}+O\left(\Delta t^{3}\right)}{\Delta t}\right) \\
& -i \partial_{t} u \\
& +\partial_{x}^{2}\left(\frac{u^{n}+\Delta t \partial_{t} u^{n}+(1 / 2) \Delta t^{2} \partial_{t}^{2} u^{n}+u^{n}+O\left(\Delta t^{3}\right)}{2}\right) \\
& -\partial_{x}^{2} u^{n}+\alpha u^{n} v^{n} \\
& -\frac{\alpha}{4}\left[\left(u^{n}+\Delta t \partial_{t} u^{n}+\left(\frac{1}{2}\right) \tau^{2} \partial_{t}^{2} u^{n}+u^{n}+O\left(\Delta t^{3}\right)\right)\right. \\
& \left.\cdot\left(v^{n}+\Delta t \partial_{t} v^{n}+\left(\frac{1}{2}\right) \Delta t^{2} \partial_{t}^{2} v^{n}+v^{n}+O\left(\Delta t^{3}\right)\right)\right] .
\end{aligned}
$$

From this result, we can deduce that $\left\|\tau_{1}^{n}\right\|_{s} \leq C \tau^{2}$. Analogously,

$$
\begin{aligned}
\tau_{2}^{n} & =\frac{v^{n}+\Delta t \partial_{t} v^{n}+(1 / 2) \Delta t^{2} \partial_{t}^{2} v^{n}-v^{n}+O\left(\Delta t^{3}\right)}{\Delta t}-\partial_{t} v^{n} \\
& -\gamma \mathscr{H} \partial_{x}^{2}\left(\frac{v^{n}+\Delta t \partial_{t} v^{n}+(1 / 2) \Delta t^{2} \partial_{t}^{2} v^{n}+v^{n}+O\left(\Delta t^{3}\right)}{2}\right) \\
& +\gamma \mathscr{H} \partial_{x}^{2} v^{n} \\
& +\beta \partial_{x}\left(\left|u^{n}\right|^{2}-\frac{\left|u^{n}+\Delta t \partial_{t} u^{n}+u^{n}+O\left(\Delta t^{3}\right)\right|^{2}}{4}\right) .
\end{aligned}
$$

As a consequence, we also have that $\left\|\tau_{2}^{n}\right\|_{s} \leq C \tau^{2}$. 
On the other hand, to estimate nonlinear terms, let us observe that

$$
\begin{aligned}
& \left\|\widehat{u}^{n} \widehat{v}^{n}-\widehat{u}_{N}^{n} \widehat{v}_{N}^{n}\right\|_{s} \leq C\left(\left\|u^{n+1}\right\|_{s}\left\|\eta_{2}^{n+1}\right\|_{s}+\left\|v_{N}^{n+1}\right\|_{s}\right. \\
& \cdot\left\|\eta_{2}^{n+1}\right\|_{s}+\left\|u^{n+1}\right\|_{s}\left\|\eta_{2}^{n}\right\|_{s}+\left\|v_{N}^{n}\right\|_{s}\left\|\eta_{1}^{n+1}\right\|_{s}+\left\|u^{n}\right\|_{s} \\
& \cdot\left\|\eta_{2}^{n+1}\right\|_{s}+\left\|v_{N}^{n+1}\right\|_{s}\left\|\eta_{1}^{n}\right\|_{s}+\left\|v^{n}\right\|_{s}\left\|\eta_{1}^{n}\right\|_{s}+\left\|u_{N}^{n}\right\|_{s} \\
& \left.\cdot\left\|\eta_{2}^{n}\right\|_{s}\right), \\
& \left\|\partial_{x}\left(\left|\widehat{u}^{n}\right|^{2}-\left|\widehat{u}_{N}\right|^{2}\right)\right\|_{s}=\| \partial_{x}\left|\frac{u^{n+1}+u^{n}}{2}\right|^{2} \\
& -\partial_{x}\left|\frac{u_{N}^{n+1}+u_{N}^{n}}{2}\right|^{2} \| \\
& \quad=\frac{1}{4} \| \partial_{x}\left[\left(\overline{u^{n+1}}+\overline{u^{n}}\right)\left(\eta_{1}^{n+1}+\eta_{1}^{n}\right)\right. \\
& \left.+\left(u_{N}^{n+1}+u_{N}^{n}\right)\left(\overline{\eta_{1}^{n+1}}+\overline{\eta_{1}^{n}}\right)\right] \|_{s} \\
& \leq C\left(\left\|\partial_{x}\left(\eta_{1}^{n+1}+\eta_{1}^{n}\right)\right\|_{s}+\left\|\eta_{1}^{n+1}+\eta_{1}^{n}\right\|_{s}\right) .
\end{aligned}
$$

Now letting $\phi=2 \widehat{\eta}_{1}^{n}$ in (64), one can get

$$
\begin{aligned}
& \left\langle i e_{1 t}^{n}, 2 \hat{\eta}_{1}^{n}\right\rangle_{s}+\left\langle\partial_{x}^{2} \widehat{e}_{1}^{n}, 2 \widehat{\eta}_{1}^{n}\right\rangle_{s}-\alpha\left\langle\widehat{u}^{n} \widehat{v}^{n}-\widehat{u}_{N}^{n} \widehat{v}_{N}^{n}, 2 \widehat{\eta}_{1}^{n}\right\rangle_{s} \\
& =\left\langle\tau_{1}^{n}, 2 \widehat{\eta}_{1}^{n}\right\rangle_{s} .
\end{aligned}
$$

We recall that $e_{1}^{n}=\xi_{1}^{n}+\eta_{1}^{n}$. Therefore, (71) leads to

$$
\begin{aligned}
\frac{i}{\Delta t} & \left(\left\|\eta_{1}^{n+1}\right\|_{s}^{2}-\left\|\eta_{1}^{n}\right\|_{s}^{2}\right) \\
= & \frac{1}{2}\left\|\partial_{x}\left(\frac{\eta_{1}^{n+1}+\eta_{1}^{n}}{2}\right)\right\|_{s}^{2}+\alpha\left\langle\widehat{u}^{n} \widehat{v}^{n}-\widehat{u}_{N}^{n} \widehat{v}_{N}^{n}, 2 \widehat{\eta}_{1}^{n}\right\rangle_{s} \\
& +\left\langle\tau_{1}^{n}, 2 \widehat{\eta}_{1}^{n}\right\rangle_{s} .
\end{aligned}
$$

Form hypothesis, $u, v \in C\left([0, T], H_{\text {per }}^{r}\right)$. For this reason, there exists a constant $B>0$ such that $\|u(t)\|_{s}+\|v(t)\|_{s} \leq B$, for all $t \in[0, T]$. Then, by taking imaginary part of $(72)$, one obtains

$$
\begin{aligned}
& \left\|\eta_{1}^{n+1}\right\|_{s}^{2}-\left\|\eta_{1}^{n}\right\|_{s}^{2} \\
& \quad \leq C \Delta t\left(\left\|\widehat{u}^{n} \widehat{v}^{n}-\widehat{u}_{N}^{n} \widehat{v}_{N}^{n}\right\|_{s}^{2}+\left\|\hat{\eta}_{1}^{n}\right\|_{s}^{2}+\left\|\tau_{1}^{n}\right\|_{s}^{2}+\left\|\widehat{\eta}_{1}^{n}\right\|_{s}^{2}\right) \\
& \quad \leq C \Delta t\left(\left\|\eta_{2}^{n+1}\right\|_{s}^{2}+\left\|\eta_{1}^{n+1}\right\|_{s}^{2}+\left\|\eta_{2}^{n}\right\|_{s}^{2}+\left\|\eta_{1}^{n}\right\|_{s}^{2}+\Delta t^{4}\right) .
\end{aligned}
$$

Thus, we arrive at

$$
\begin{aligned}
& \left\|\eta_{1}^{n+1}\right\|_{s}^{2}(1-C \Delta t) \\
& \quad \leq C \Delta t\left(\left\|\eta_{2}^{n}\right\|_{s}^{2}+\left\|\eta_{2}^{n+1}\right\|_{s}^{2}+\Delta t^{4}\right)+(1+C \Delta t)\left\|\eta_{1}^{n}\right\|_{s}^{2}, \\
& \left\|\eta_{1}^{n+1}\right\|_{s}^{2} \\
& \leq \frac{1+C \Delta t}{1-C \Delta t}\left\|\eta_{1}^{n}\right\|_{s}^{2} \\
& \quad+\frac{C \Delta t}{1-C \Delta t}\left(\left\|\eta_{2}^{n+1}\right\|_{s}^{2}+\left\|\eta_{2}^{n}\right\|_{s}^{2}+\Delta t^{4}\right) .
\end{aligned}
$$

As a consequence of this,

$$
\left\|\eta_{1}^{n+1}\right\|_{s}^{2} \leq C\left\|\eta_{1}^{n}\right\|_{s}^{2}+C \Delta t\left(\left\|\eta_{2}^{n+1}\right\|_{s}^{2}+\left\|\eta_{2}^{n}\right\|_{s}^{2}\right)+C \Delta t^{5} .
$$

Then summing up the previous equation for $n=0$ to $m$ and taking into account that $n=T / \Delta t$, it follows that

$$
\begin{aligned}
\sum_{n=0}^{m}\left\|\eta_{1}^{n+1}\right\|_{s}^{2} \leq & C \sum_{n=0}^{m}\left\|\eta_{1}^{n}\right\|_{s}^{2}+C \Delta t \sum_{n=0}^{m}\left(\left\|\eta_{2}^{n+1}\right\|_{s}^{2}+\left\|\eta_{2}^{n}\right\|_{s}^{2}\right) \\
& +C \Delta t^{4} .
\end{aligned}
$$

Therefore,

$$
\left\|\eta_{1}^{m+1}\right\|_{s}^{2} \leq C\left(\left\|\eta_{1}^{0}\right\|_{s}^{2}+\Delta t \sum_{n=0}^{m+1}\left\|\eta_{2}^{n}\right\|_{s}^{2}+\Delta t^{4}\right) .
$$

Analogously, letting $\psi=2 \widehat{\eta}_{2}^{n}$ in (65), one can get

$$
\begin{aligned}
& \left\langle e_{2 t}^{n}, 2 \widehat{\eta}_{2}^{n}\right\rangle_{s}-\gamma\left\langle\mathscr{H} \partial_{x}^{2} \widehat{e}_{2}^{n}, 2 \widehat{\eta}_{2}^{n}\right\rangle_{s} \\
& \quad-\beta\left\langle\partial_{x}\left(\left|\hat{u}^{n}\right|^{2}-\left|\widehat{u}_{N}^{n}\right|^{2}\right), 2 \widehat{\eta}_{2}\right\rangle_{s}=\left\langle\tau_{2}^{n}, 2 \widehat{\eta}_{2}^{n}\right\rangle_{s} .
\end{aligned}
$$

We recall that $e_{2}^{n}=\xi_{2}^{n}+\eta_{2}^{n}$. Therefore, (78) leads to

$$
\begin{aligned}
& \frac{1}{\Delta t}\left(\left\|\eta_{2}^{n+1}\right\|_{s}^{2}-\left\|\eta_{2}^{n}\right\|_{s}^{2}\right) \\
& \quad=\beta\left\langle\partial_{x}\left(\left|\hat{u}^{n}\right|^{2}-\left|\widehat{u}_{N}^{n}\right|^{2}\right), 2 \hat{\eta}_{2}^{n}\right\rangle_{s}+\left\langle\tau_{2}^{n}, 2 \widehat{\eta}_{2}^{n}\right\rangle_{s} .
\end{aligned}
$$

Then, by taking imaginary part of (79), one obtains

$$
\begin{gathered}
\left\|\eta_{2}^{n+1}\right\|_{s}^{2}-\left\|\eta_{2}^{n}\right\|_{s}^{2} \leq C \Delta t\left(\left\|\partial_{x}\left(\left|\hat{u}^{n}\right|^{2}-\left|\hat{u}_{N}^{n}\right|^{2}\right)\right\|_{s}\left\|\hat{\eta}_{2}^{n}\right\|_{s}\right. \\
\left.+\left\|\tau_{2}^{n}\right\|_{s}\left\|\hat{\eta}_{2}^{n}\right\|_{s}\right) \leq C \Delta t\left(\left\|\partial_{x} \eta_{1}^{n+1}\right\|_{s}^{2}+\left\|\partial_{x} \eta_{1}^{n}\right\|_{s}^{2}\right. \\
\left.+\left\|\eta_{2}^{n+1}\right\|_{s}^{2}+\left\|\eta_{1}^{n+1}\right\|_{s}^{2}+\left\|\eta_{1}^{n}\right\|_{s}^{2}+\left\|\eta_{2}^{n}\right\|_{s}^{2}+\Delta t^{4}\right) .
\end{gathered}
$$

Thus, we arrive at

$$
\begin{gathered}
\left\|\eta_{2}^{n+1}\right\|_{s}^{2}(1-C \Delta t) \leq C \Delta t\left(\left\|\partial_{x} \eta_{1}^{n+1}\right\|_{s}^{2}+\left\|\partial_{x} \eta_{1}^{n}\right\|_{s}^{2}\right. \\
\left.+\left\|\eta_{1}^{n+1}\right\|_{s}^{2}+\left\|\eta_{1}^{n}\right\|_{s}^{2}+\Delta t^{4}\right)+(1+C \Delta t)\left\|\eta_{2}^{n}\right\|_{s}^{2}, \\
\left\|\eta_{2}^{n+1}\right\|_{s}^{2} \leq \frac{1+C \Delta t}{1-C \Delta t}\left\|\eta_{2}^{n}\right\|_{s}^{2}+\frac{C \Delta t}{1-C \Delta t}\left(\left\|\partial_{x} \eta_{1}^{n+1}\right\|_{s}^{2}\right. \\
\left.+\left\|\partial_{x} \eta_{1}^{n}\right\|_{s}^{2}+\left\|\eta_{1}^{n+1}\right\|_{s}^{2}+\left\|\eta_{1}^{n}\right\|_{s}^{2}+\Delta t^{4}\right) .
\end{gathered}
$$


As a consequence of this,

$$
\begin{aligned}
& \left\|\eta_{2}^{n+1}\right\|_{s}^{2} \\
& \leq C\left\|\eta_{2}^{n}\right\|_{s}^{2} \\
& \quad+C \Delta t\left(\left\|\partial_{x} \eta_{1}^{n+1}\right\|_{s}^{2}+\left\|\partial_{x} \eta_{1}^{n}\right\|_{s}^{2}+\left\|\eta_{1}^{n+1}\right\|_{s}^{2}+\left\|\eta_{1}^{n}\right\|_{s}^{2}\right) \\
& \quad+C \Delta t^{5} .
\end{aligned}
$$

Then, summing up the previous equation for $n=0$ to $m$, it follows that

$$
\begin{aligned}
& \sum_{n=0}^{m}\left\|\eta_{2}^{n+1}\right\|_{s}^{2} \leq C \sum_{n=0}^{m}\left\|\eta_{2}^{n}\right\|_{s}^{2} \\
& \quad+C \Delta t \sum_{n=0}^{m}\left(\left\|\partial_{x} \eta_{1}^{n+1}\right\|_{s}^{2}+\left\|\partial_{x} \eta_{1}^{n}\right\|_{s}^{2}+\left\|\eta_{1}^{n+1}\right\|_{s}^{2}+\left\|\eta_{1}^{n}\right\|_{s}^{2}\right) \\
& \quad+C \Delta t^{4}
\end{aligned}
$$

Therefore,

$$
\begin{aligned}
& \left\|\eta_{2}^{m+1}\right\|_{s}^{2} \\
& \leq C\left(\left\|\eta_{2}^{0}\right\|_{s}^{2}+\Delta t \sum_{n=0}^{m+1}\left\|\partial_{x} \eta_{1}^{n}\right\|_{s}^{2}+\Delta t \sum_{n=0}^{m+1}\left\|\eta_{1}^{n}\right\|_{s}^{2}+\Delta t^{4}\right) .
\end{aligned}
$$

In order to estimate the terms $\left\|\partial_{x} \eta_{1}^{n}\right\|_{s}$, let $\phi=\eta_{1 t}^{n}$ in (64) to get

$$
\left\langle i e_{1 t}^{n}+\partial_{x}^{2} \widehat{e}_{1}^{n}-\alpha\left(\widehat{u}^{n} \widehat{v}^{n}-\widehat{u}_{N}^{n} \widehat{v}_{N}^{n}\right), \eta_{1 t}^{n}\right\rangle_{s}=\left\langle\tau_{1}^{n}, \eta_{1 t}^{n}\right\rangle_{s} .
$$

Taking imaginary part of the resulting equation, one deduces that

$$
\left\|\eta_{1 t}^{n}\right\|_{s}^{2}=\alpha \operatorname{Im}\left\langle\widehat{u}^{n} \widehat{v}^{n}-\widehat{u}_{N}^{n} \widehat{v}_{N}^{n}, \eta_{1 t}^{n}\right\rangle_{s}+\operatorname{Im}\left\langle\tau_{1}^{n}, \eta_{1 t}^{n}\right\rangle_{s} .
$$

Therefore,

$$
\begin{aligned}
\left\|\eta_{1 t}^{n}\right\|_{s}^{2} \leq & C\left(\left\|\eta_{2}^{n+1}\right\|_{s}^{2}+\left\|\eta_{1}^{n+1}\right\|_{s}^{2}+\left\|\eta_{2}^{n}\right\|_{s}^{2}+\left\|\eta_{1}^{n}\right\|_{s}^{2}\right) \\
& +C \Delta t^{4} .
\end{aligned}
$$

Letting $\psi=2 \eta_{1 t}^{n}$ in (64),

$$
\begin{aligned}
& \left\langle i e_{1 t}^{n}, 2 \eta_{1 t}^{n}\right\rangle_{s}+\left\langle\partial_{x}^{2} \widehat{e}_{1}^{n}, 2 \eta_{1 t}^{n}\right\rangle_{s} \\
& =\alpha\left\langle\widehat{u}^{n} \widehat{v}^{n}-\widehat{u}_{N}^{n} \widehat{v}_{N}^{n}, 2 \eta_{1 t}^{n}\right\rangle_{s}+\left\langle\tau_{1}^{n}, 2 \eta_{1 t}^{n}\right\rangle_{s},
\end{aligned}
$$

and thus, simplifying the equation above and taking real part of the resulting equation, we obtain that

$$
\begin{aligned}
- & \frac{1}{\Delta t}\left(\left\|\partial_{x} \eta_{1}^{n+1}\right\|_{s}^{2}-\left\|\partial_{x} \eta_{1}^{n}\right\|_{s}^{2}\right) \\
& =2 \alpha \operatorname{Re}\left\langle\widehat{u}^{n} \widehat{v}^{n}-\widehat{u}_{N}^{n} \widehat{v}_{N}^{n}, \eta_{1 t}^{n}\right\rangle_{s}+2 \operatorname{Re}\left\langle\tau_{1}^{n}, \eta_{1 t}^{n}\right\rangle_{s} .
\end{aligned}
$$

Therefore, using the estimate for $\left\|\eta_{1 t}\right\|_{s}$ obtained above, one realizes that

$$
\begin{aligned}
& \frac{1}{\Delta t}\left(\left\|\partial_{x} \eta_{1}^{n+1}\right\|_{s}^{2}-\left\|\partial_{x} \eta_{1}^{n}\right\|_{s}^{2}\right) \\
& \leq C\left(\left\|\eta_{1} n+1\right\|_{s}^{2}+\left\|\eta_{1}^{n}\right\|^{2}+\left\|\eta_{2}^{n+1}\right\|_{s}^{2}+\left\|\eta_{2}^{n}\right\|_{s}^{2}+\left\|\eta_{1 t}^{n}\right\|_{s}^{2}\right) \\
& \quad+C \Delta t^{4} \\
& \leq C\left(\left\|\eta_{1}^{n+1}\right\|_{s}^{2}+\left\|\eta_{1}^{n}\right\|_{s}^{2}+\left\|\eta_{2}^{n+1}\right\|_{s}^{2}+\left\|\eta_{2}^{n}\right\|_{s}^{2}\right)+C \Delta t^{4} .
\end{aligned}
$$

Summing up $n=0, \ldots, m$, we arrive at

$$
\begin{aligned}
\left\|\partial_{x} \eta_{1}^{m+1}\right\|_{s}^{2} \leq & C\left(\left\|\partial_{x} \eta_{1}^{0}\right\|_{s}^{2}+\Delta t \sum_{n=0}^{m+1}\left(\left\|\eta_{1}^{n}\right\|_{s}^{2}+\left\|\eta_{2}^{n}\right\|_{s}^{2}\right)\right) \\
& +C \Delta t^{4} .
\end{aligned}
$$

We have from the previous results that

$$
\begin{aligned}
& E^{m}:=\left\|\eta_{1}^{m+1}\right\|_{s}^{2}+\left\|\partial_{x} \eta_{1}^{m+1}\right\|_{s}^{2}+\left\|\eta_{2}^{m+1}\right\|_{s}^{2}=\left\|\eta_{1}^{m+1}\right\|_{s+1}^{2} \\
& +\left\|\eta_{2}^{m+1}\right\|_{s}^{2} \leq C\left(\left\|\eta_{1}^{0}\right\|_{s}^{2}+\Delta t \sum_{n=0}^{m+1}\left\|\eta_{2}^{n}\right\|_{s}^{2}+\Delta t^{4}\right) \\
& +C\left(\left\|\eta_{2}^{0}\right\|_{s}^{2}+\Delta t \sum_{n=0}^{m+1}\left\|\partial_{x} \eta_{1}^{n}\right\|_{s}^{2}+\Delta t \sum_{n=0}^{m+1}\left\|\eta_{1}^{n}\right\|_{s}^{2}\right. \\
& \left.+\Delta t^{4}\right)+C\left(\left\|\partial_{x} \eta_{1}^{0}\right\|_{s}^{2}+\Delta t \sum_{n=0}^{m+1}\left(\left\|\eta_{1}^{n}\right\|_{s}^{2}+\left\|\eta_{2}^{n}\right\|_{s}^{2}\right)\right. \\
& \left.+\Delta t^{4}\right) \leq C\left(\left\|\eta_{1}^{0}\right\|_{s}^{2}+\left\|\partial_{x} \eta_{1}^{0}\right\|_{s}^{2}+\left\|\eta_{2}^{0}\right\|_{s}^{2}\right. \\
& \left.+\Delta t \sum_{n=0}^{m}\left(\left\|\eta_{1}^{n}\right\|_{s}^{2}+\left\|\eta_{2}^{n}\right\|_{s}^{2}+\left\|\partial_{x} \eta_{1}^{n}\right\|_{s}^{2}\right)+\Delta t^{4}\right) \\
& +C \Delta t\left\|\partial_{x} \eta_{1}^{m+1}\right\|_{s}^{2}+C \Delta t\left\|\eta_{1}^{m+1}\right\|_{s}^{2}+C \Delta t\left\|\eta_{2}^{m+1}\right\|_{s}^{2}
\end{aligned}
$$

We have that

$$
\begin{aligned}
& E^{m}(1-C \Delta t) \leq C\left(\left\|\eta_{1}^{0}\right\|_{s}^{2}+\left\|\partial_{x} \eta_{1}^{0}\right\|_{s}^{2}+\left\|\eta_{2}^{0}\right\|_{s}^{2}\right. \\
& \left.+\Delta t \sum_{n=0}^{m}\left[\left\|\eta_{1}^{n}\right\|_{s}^{2}+\left\|\eta_{2}^{n}\right\|_{s}^{2}+\left\|\partial_{x} \eta_{1}^{n}\right\|_{s}^{2}\right]+\Delta t^{4}\right), \\
& E^{m} \leq C\left(\left\|\eta_{1}^{0}\right\|_{s+1}^{2}+\left\|\eta_{2}^{0}\right\|_{s}^{2}\right)+C \Delta t^{4}+C \Delta t \sum_{n=1}^{m} E^{n-1} .
\end{aligned}
$$

Finally, since $\left\|\eta_{1}^{0}\right\|_{s}=\left\|\eta_{2}^{0}\right\|_{s}=0$,

$$
E^{m} \leq C \Delta t^{4}+C \Delta t \sum_{n=1}^{m} E^{n-1},
$$

and the result follows from Gronwall's lemma. 


\section{Numerical Experiments}

The purpose of this section is to present some numerical simulations using the numerical scheme described in the previous section. We point out that the technique and procedure used here for the numerical simulations are similar to the work by Muñoz Grajales in [15] about a Fourier-Galerkin numerical scheme applied to a $1 \mathrm{D}$ Benney-Luke-Paumond equation.

We recall that any function in $S_{N}$ can be written as

$$
u=\sum_{j=-N / 2}^{N / 2} \widehat{u}_{j} \phi_{j},
$$

with

$$
\widehat{u}_{j}=\frac{1}{L} \int_{0}^{L} u(x) \overline{\phi_{j}(x)} d x
$$

As a consequence, we have that scheme (57) can be written equivalently as

$$
\begin{aligned}
i \frac{\widehat{u}_{j}^{n+1}-\widehat{u}_{j}^{n}}{\Delta t}= & w_{j}^{2}\left(\frac{\widehat{u}_{j}^{n+1}+\widehat{u}_{j}^{n}}{2}\right) \\
& +\frac{\alpha}{2}\left(P_{j}\left[u^{(n+1)} v^{(n+1)}\right]+P_{j}\left[u^{(n)} v^{(n)}\right]\right),
\end{aligned}
$$

$$
\begin{aligned}
\frac{\widehat{v}_{j}^{n+1}-\widehat{v}_{j}^{n}}{\Delta t}= & -\gamma i w_{j}\left|w_{j}\right|\left(\frac{\widehat{v}_{j}^{n+1}+\widehat{v}_{j}^{n}}{2}\right) \\
& +\frac{\beta i w_{j}}{2}\left(P_{j}\left[\left|u^{(n+1)}\right|^{2}\right]+P_{j}\left[\left|u^{(n)}\right|^{2}\right]\right),
\end{aligned}
$$

subject to $\widehat{u}_{j}^{0}=\widehat{u}_{0 j} \widehat{v}_{j}^{0}=\widehat{v}_{0 j}$ and where

$$
w_{j}=\frac{2 \pi j}{L}, \quad j=-\frac{N}{2}, \ldots, 0, \ldots, \frac{N}{2},
$$

and $P_{j}[\cdot]$ denotes the operator

$$
P_{j}[g]=\frac{1}{L} \int_{0}^{L} g(x) e^{-i w_{j} x} d x .
$$

Additionally, let $u^{(n)}, v^{(n)}$ be the approximations of the unknowns $u(x, t), v(x, t)$, respectively, at time $t_{n}=n \Delta t$, where $\Delta t$ represents the time step of the method and $\widehat{u}_{j}^{n}, \widehat{v}_{j}^{n}$ are the approximations to the Fourier transforms of the functions $u$ and $v$, respectively, with respect to the variable $x$, evaluated at the time $n \Delta t$.

Observe that, in the scheme given in (97), the unknowns $\widehat{u}_{j}^{n+1}, \widehat{v}_{j}^{n+1}$ and $u^{(n+1)}, v^{(n+1)}$ at $t_{n}=(n+1) \Delta t$ must be computed by iteration. To do this, we use the iterative process

$$
\begin{aligned}
& \widehat{u}_{j}^{n+1, k+1}=\frac{\widehat{u}_{j}^{n}-\Delta t i w_{j}^{2} \widehat{u}_{j}^{n} / 2-(\Delta t i \alpha / 2)\left(P_{j}\left[u^{n+1, k} v^{n+1, k}\right]+P_{j}\left[u^{(n)} v^{(n)}\right]\right)}{1+\Delta t i w_{j}^{2} / 2}, \\
& \widehat{v}_{j}^{n+1, k+1}=\frac{\widehat{v}_{j}^{n}-\Delta t \gamma i w_{j}\left|w_{j}\right| \hat{v}_{j}^{n} / 2+\left(\Delta t i w_{j} \beta / 2\right)\left(P_{j}\left[\left|u^{n+1, k}\right|^{2}\right]+P_{j}\left[\left|u^{(n)}\right|^{2}\right]\right)}{1+\Delta t i \gamma w_{j}^{2} / 2}, \quad k=1,2, \ldots
\end{aligned}
$$

To compute the initial values $\widehat{u}_{j}^{n+1,0}, \widehat{v}_{j}^{n+1,0}, u^{n+1,0}$, and $v^{n+1,0}$ for this iteration, we use the explicit scheme

$$
\begin{aligned}
& i \frac{\widehat{u}_{j}^{n+1,0}-\widehat{u}_{j}^{n}}{\Delta t}=w_{j}^{2} \widehat{u}_{j}^{n}+\alpha P_{j}\left[u^{(n)} v^{(n)}\right], \\
& \frac{\widehat{v}_{j}^{n+1,0}-\widehat{v}_{j}^{n}}{\Delta t}=-\gamma i w_{j}\left|w_{j}\right| \hat{v}_{j}^{n}+\beta i w_{j} P_{j}\left[\left|u^{(n)}\right|^{2}\right] .
\end{aligned}
$$

In all of the numerical experiments presented, we used double precision in Matlab R2016b on a Mac platform. The approximation of the Fourier-type integral appearing in the definition of the operator $P_{j}[\cdot]$ is performed through the wellknown Fast Fourier Transform (FFT) algorithm.

5.1. Convergence Rate in Space. To verify the numerical properties of the fully discrete scheme proposed in the present paper, we use a technique similar to that in [15]. In particular, we want to validate the spectral order of convergence in space of the Crank-Nicholson Fourier-Galerkin numerical scheme considered. In Figure 1, we present a simulation where a small time step $\Delta t=1 e-5$ is fixed, $L=20$, and the number of points in space is gradually increased. We use the analytical travelling wave solution of the SBO system for $\gamma=0$, given by

$$
\begin{aligned}
& u(x, t)=A e^{i w_{p} t} e^{i c(x-c t) / 2} \operatorname{sech}\left(B\left(x-c t-a_{0}\right)\right), \\
& v(x, t)=-\frac{A^{2} \beta}{c} \operatorname{sech}^{2}\left(B\left(x-c t-a_{0}\right)\right),
\end{aligned}
$$

where

$$
\begin{aligned}
& A=\sqrt{\frac{2 c}{\alpha \beta}\left(w_{p}-\frac{c^{2}}{4}\right)}, \\
& B=\sqrt{w_{p}-\frac{c^{2}}{4}},
\end{aligned}
$$


with $\alpha=\beta=1, c=0.5, w_{p}=1.5$, and $a_{0}=10$. We start with $N=2^{6}$ and then $N$ is increased by 2 until we get $N=2^{9}$. For every value of $N$, we compute the numerical solution until reaching the time $t=1$. From the results in Figure 1, we see that the fully discrete method (57) has spectral accuracy in space (as established in Theorem 2), and the error decreases very rapidly approximately as $N^{-6.07}$. We point out that this decay rate is faster than that in pure finite difference methods.

5.2. Convergence Rate in Time. In this section, we wish to validate numerically the rate of convergence in time for the numerical scheme (57). This numerical simulation is performed using the solitary wave solution (102) with the same model's parameters as in the previous numerical experiment. Furthermore, we set $N=2^{12}(\Delta x=L / N \approx 5 e-3)$ with the objective that the error in space does not dominate the total error. By starting with $\Delta t=1 / 2^{2}$ and decreasing the time step by $1 / 2$ until $\Delta t=1 / 2^{6}$, the numerical solution is computed until reaching $t=1$. The results are presented in Figure 2, from where we can observe that the error in time of the numerical scheme is of order 2 , in perfect agreement with Theorem 2.

5.3. Numerical Results for the Full SBO System. Finally, we illustrate the numerical scheme (57) in the case that $\gamma=1 \neq 0$, where no analytical solution is available in the literature. We use the approximation to a travelling wave solution of the $\mathrm{SBO}$ system given by

$$
\begin{aligned}
& u(x, t)=e^{i w_{p} t} e^{i c(x-c t) / 2} \phi(x-c t), \\
& v(x, t)=\psi(x-c t),
\end{aligned}
$$

with $\phi, \psi$ being periodic real-valued functions computed through a Newton-iterative procedure, together with a collocation-Fourier method applied to the system

$$
\begin{aligned}
\phi^{\prime \prime}+\left(-w_{p}+\frac{c^{2}}{4}\right) \phi & =\alpha \phi \psi, \\
-\gamma \mathscr{H}\left(\psi^{\prime}\right)-c \psi & =\beta \phi^{2},
\end{aligned}
$$

for the parameters $\alpha=\beta=\gamma=1, c=0.5$, and $w_{p}=1.5$. In Figure 3, we show the plot of the approximate functions $\phi, \psi$, with period $L=4 \pi / c$ obtained by using Newton's procedure mentioned above, with starting point

$$
\begin{aligned}
& \phi_{0}(x)=2 \cos \left(\frac{2 \pi x}{L}\right), \\
& \psi_{0}(x)=\cos \left(\frac{2 \pi x}{L}\right) .
\end{aligned}
$$

We run the numerical solver (57) using the approximate travelling wave (104) at $t=0$ as initial data and numerical parameters $N=2^{9}, \Delta t=1 e-3$, and $L=4 \pi / c \approx 25.13$. The result is presented in Figure 4. We observe that the numerical and the expected profile coincide at $t=5$ with a maximum error of $2 e-4$, showing that the scheme proposed

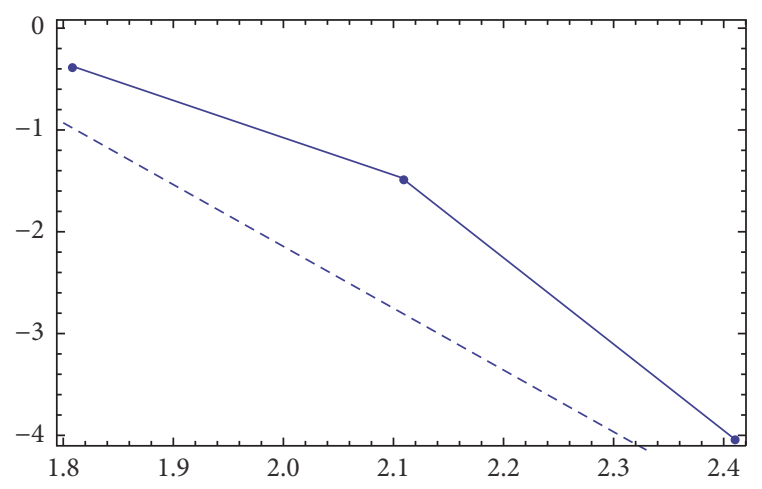

FIGURE 1: Plot of the decimal logarithm of the maximum error against $\log _{10} N$. The time step is fixed at $\Delta t=1 e-5$. We see that the plot is approximately a line with slope -6.07 .

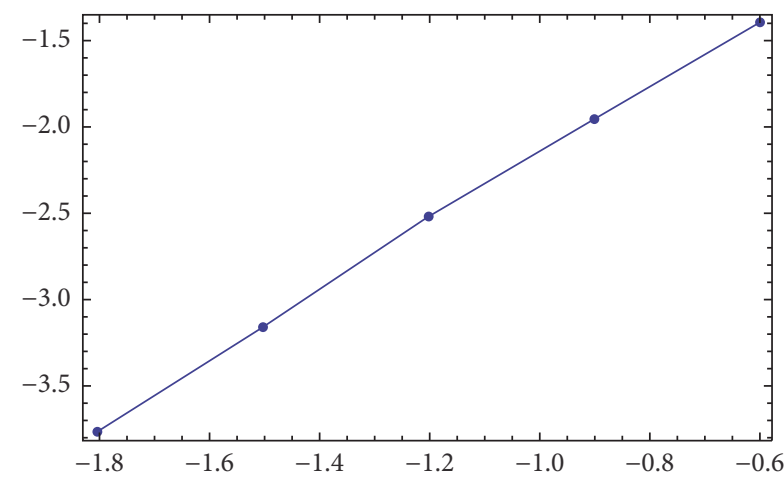

Figure 2: Plot of the decimal logarithm of the maximum error against $\log _{10} \Delta t$. The number of points in space is fixed at $N=2^{12}$. We see that the plot is approximately a line with slope 2 .

captures the nonlinear and dispersive characteristics of the solutions of the SBO system (1). Other numerical experiments with travelling wave solutions of the full SBO system were performed obtaining analogous results.

5.4. Checking Energy Conservation. It is easy to see that the quantity

$$
E(t)=\int_{0}^{L}|u(x, t)|^{2} d x
$$

must be conserved in time for the function $u(x, t)$ in system (1). In the numerical experiment presented in Figure 5, we corroborate that the fully discrete scheme (57) further conserves approximately the discrete version of $E(t)$ given by

$$
C(t)=\Delta x \sum_{k=0}^{N-1}|u(k \Delta x, t)|^{2} .
$$

The initial conditions are given by

$$
u(x, 0)=v(x, 0)=\cos (\pi x),
$$



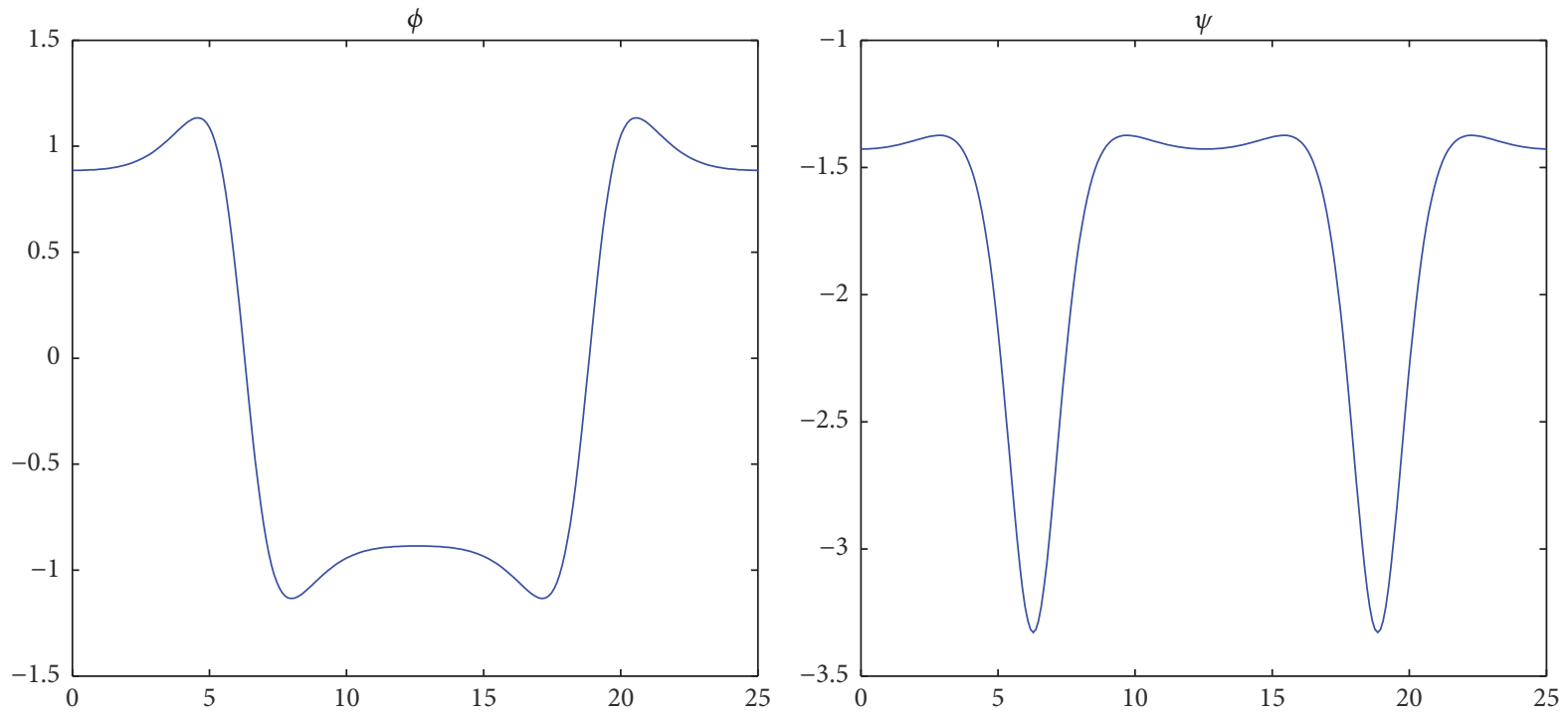

Figure 3: Approximations of the functions $\phi, \psi$ in the travelling wave solution (104).

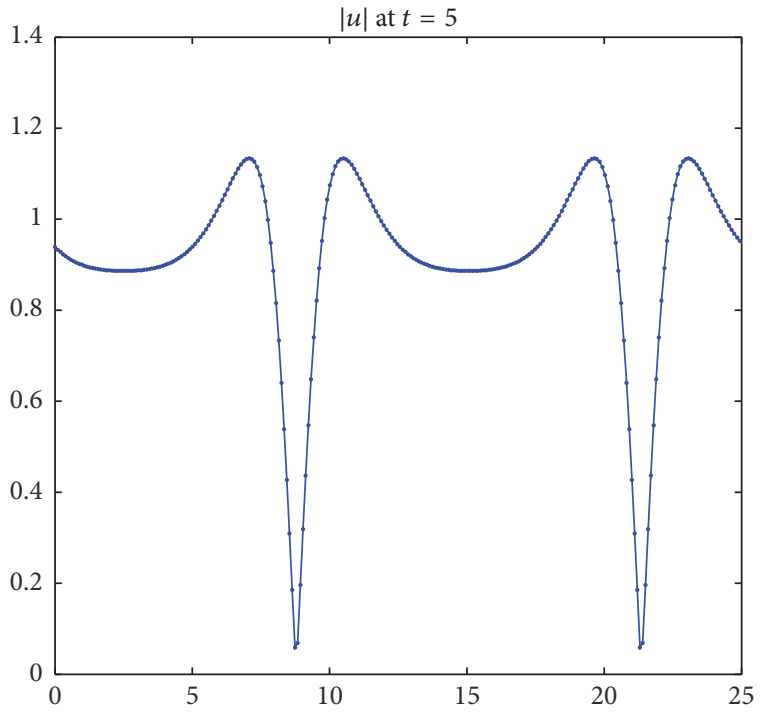

$(x)$

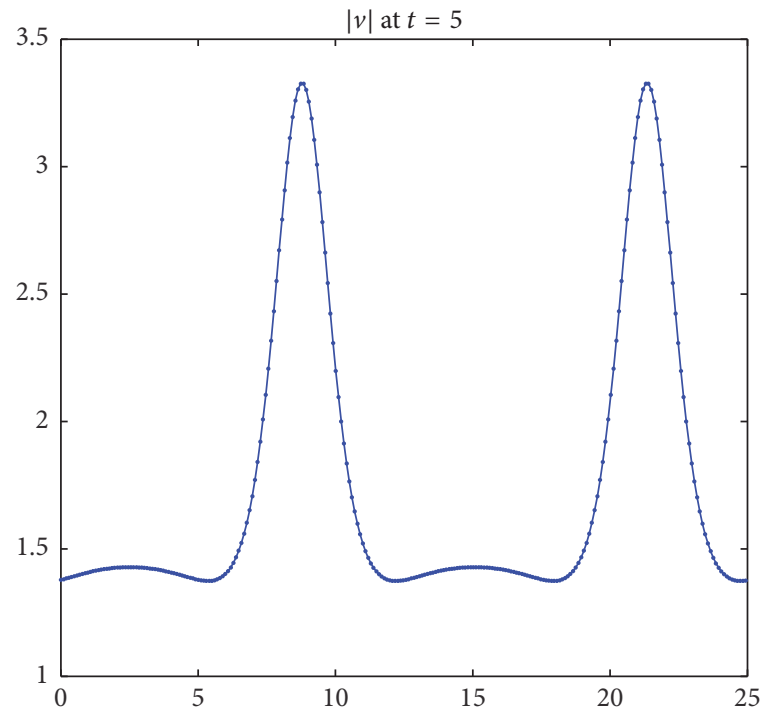

$(x)$

FIGURE 4: Evolution of a travelling wave solution of the SBO system at $t=5$. In solid line, $|u|,|v|$ computed with the numerical solver (57). In pointed line, $|u|,|v|$ for the approximate travelling wave solution (104).

and the period is $L=2$. We set other numerical parameters as $\alpha=\beta=\gamma=1$ and $\Delta t=1 e-3$, and the number of FFT points is $N=2^{8}$. Other numerical simulations conducted using the fully discrete scheme (57) with different initial conditions showed similar results.

\section{Conclusions}

In this paper, we developed a rigorous analysis of the error of the semidiscrete and fully discrete formulations of a FourierGalerkin scheme to approximate solutions of the SBO system (1). The time-stepping method was implemented by using a second-order implicit Crank-Nicholson strategy. To the best of our knowledge, a complete error analysis of a fully discrete scheme for the SBO system in the general case $\gamma \neq 0$ has not been developed in previous works. The resulting accuracy and convergence rate $O\left(N^{s+2-r}+\Delta t^{2}\right)(r>s+2)$ of the numerical solver considered were illustrated by using a family of exact solitary wave solutions of system (1) with $\gamma=0$. Numerical experiments with the complete SBO system with $\gamma \neq 0$ were also presented by using some approximations of solitary wave solutions computed using a Newton-collocation scheme combined with a collocation-Fourier method.

\section{Competing Interests}

The author declares that there is no conflict of interests regarding the publication of this paper. 


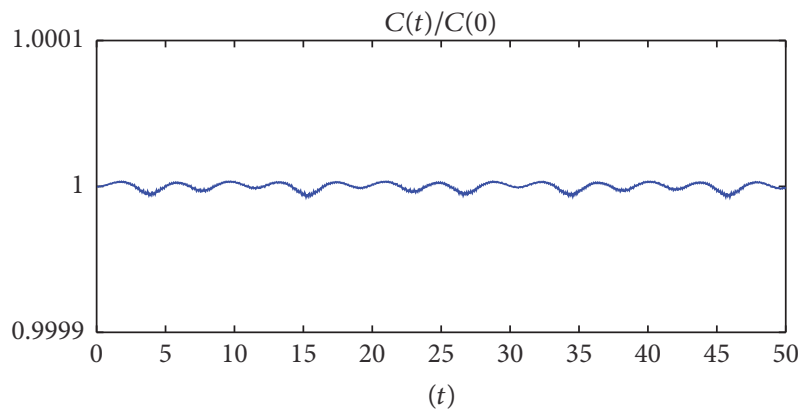

FIGURE 5: In this numerical experiment we see that the quantity $C(t)$ is close to 1 , as long as time evolves. This shows that the numerical solver (57) conserves approximately the $L^{2}$-norm of the function $u(x, t)$ in system $(1)$.

\section{Acknowledgments}

This research was supported by Departamento de Matemáticas, Universidad del Valle, Calle 13, Nro. 10000, Cali, Colombia, under Research Project C.I. 71020.

\section{References}

[1] M. Funakoshi and M. Oikawa, "The resonant interaction between a long internal gravity wave and a surface gravity wave packet," Journal of the Physical Society of Japan, vol. 52, no. 6, pp. 1982-1995, 1983.

[2] V. I. Karpman, "On the dynamics of sonic-langmuir solitons," Physica Scripta, vol. 11, no. 5, pp. 263-265, 1975.

[3] V. Djordjevic and L. Redekoop, "On two-dimensional packet of capillary-gravity waves," Journal of Fluid Mechanics, vol. 79, pp. 703-714, 1977.

[4] R. H. Grimshaw, "The modulation of an internal gravity-wave packet, and the resonance with the mean motion," Studies in Applied Mathematics, vol. 56, no. 3, pp. 241-266, 1977.

[5] D. J. Benney, "Significant interactions between small and large scale surface waves," Studies in Applied Mathematics, vol. 55, no. 2, pp. 93-106, 1976.

[6] D. J. Benney, "A general theory for interactions between short and long waves," Studies in Applied Mathematics, vol. 56, no. 1, pp. 81-94, 1977.

[7] J. Angulo and J. F. Montenegro, "Existence and evenness of solitary-wave solutions for an equation of short and long dispersive waves," Nonlinearity, vol. 13, no. 5, pp. 1595-1611, 2000.

[8] P.-L. Lions, "The concentration-compactness principle in the calculus of variations. The locally compact case. Part 1," Annales De L Institut Henri Poincaré-Analyse Non Linéare, vol. 1, no. 2, pp. 109-145, 1984.

[9] P.-L. Lions, "The concentration-compactness principle in the calculus of variations. The locally compact case. II," Annales de l'Institut Henri Poincaré. Analyse Non Linéaire, vol. 1, no. 4, pp. 223-283, 1984.

[10] J. Angulo, "Stability of solitary wave solutions for equations of short and long dispersive waves," Electronic Journal of Differential Equations, no. 72, pp. 1-8, 2006.

[11] D. Bekiranov, T. Ogawa, and G. Ponce, "Interaction equations for short and long dispersive waves," Journal of Functional Analysis, vol. 158, no. 2, pp. 357-388, 1998.
[12] H. Pecher, "Rough solutions of a Schrödinger-Benjamin-Ono system," Differential and Integral Equations. An International Journal for Theory \& Applications, vol. 19, no. 5, pp. 517-535, 2006.

[13] J. Angulo, C. Matheus, and D. Pilod, "Global well-posedness and non-linear stability of periodic traveling waves for a Schrödinger-Benjamin-Ono system," Communications on Pure and Applied Analysis, vol. 8, no. 3, pp. 815-844, 2009.

[14] J. C. Muñoz Grajales, "Error estimates for a Galerkin numerical scheme applied to a generalized BBM equation," Applicable Analysis, vol. 94, no. 7, pp. 1405-1419, 2015.

[15] J. C. Muñoz Grajales, "Analysis of a fourier-galerkin numerical scheme for a $1 \mathrm{D}$ benney-luke-paumond equation," Revista Colombiana de Matemáticas, vol. 49, no. 2, pp. 213-234, 2015.

[16] D. C. Antonopoulos and V. A. Dougalis, "rror estimates for Galerkin approximations of the 'classical' Boussinesq system," Mathematics and Computers in Simulation, vol. 82, no. 6, pp. 984-1007, 2012.

[17] D. C. Antonopoulos, V. A. Dougalis, and D. E. Mitsotakis, "Numerical solution of Boussinesq systems of the Bona-Smith family," Applied Numerical Mathematics, vol. 60, no. 4, pp. 314336, 2010.

[18] B. Pelloni and V. A. Dougalis, "Error estimates for a fully discrete spectral scheme for a class of nonlinear, nonlocal dispersive wave equations," Applied Numerical Mathematics, vol. 37, no. 12, pp. 95-107, 2001.

[19] A. Rashid and S. Akram, "Convergence of Fourier spectral method for resonant long-short nonlinear wave interaction," Applications of Mathematics, vol. 55, no. 4, pp. 337-350, 2010.

[20] Y. Maday and A. Quarteroni, "Error analysis for spectral approximation of the Korteweg-de Vries equation," RAIROModélisation Mathématique et Analyse Numérique, vol. 22, no. 3, pp. 499-529, 1988.

[21] B. Mercier, An Introduction to the Numerical Analysis of Spectral Methods, vol. 318 of Lecture Notes in Physics, Springer, New York, NY, USA, 1983. 


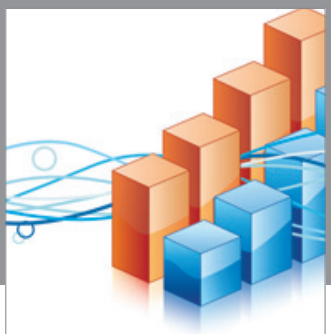

Advances in

Operations Research

vatem alat4

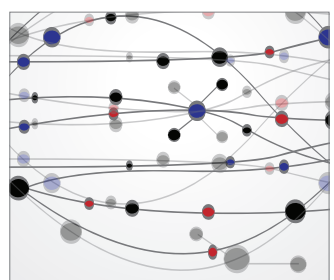

\section{The Scientific} World Journal
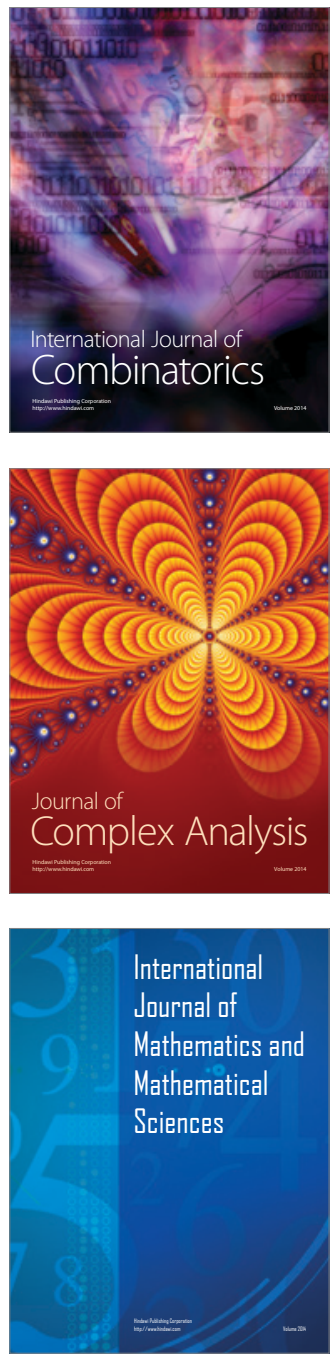
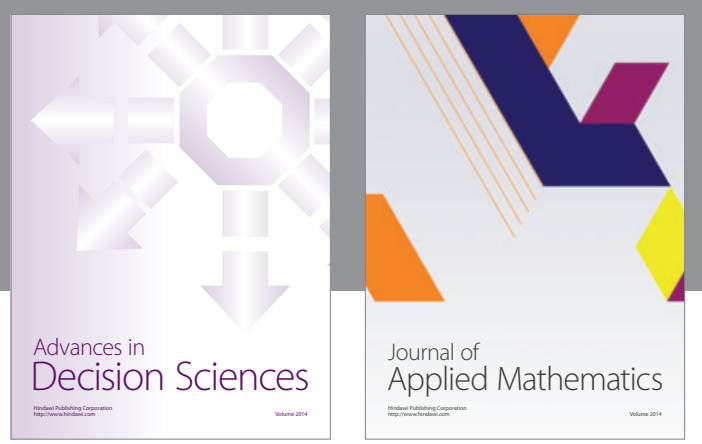

Algebra

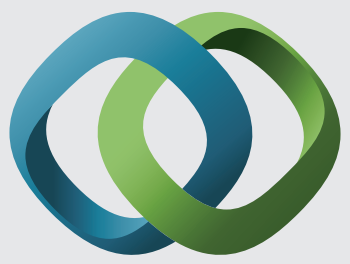

\section{Hindawi}

Submit your manuscripts at

http://www.hindawi.com
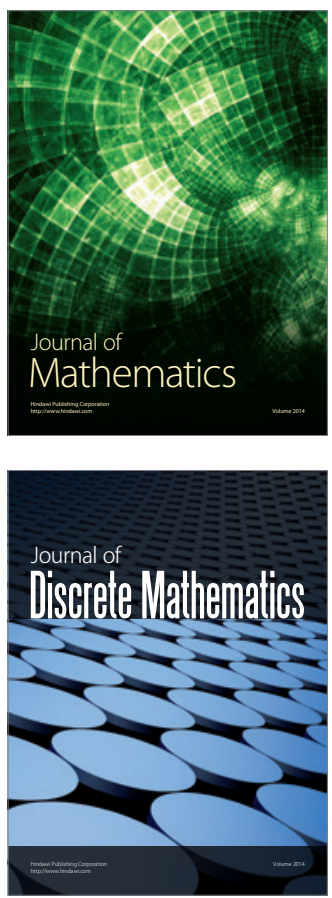

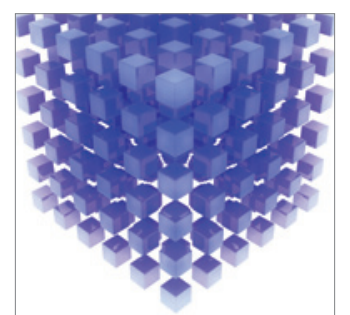

Mathematical Problems in Engineering
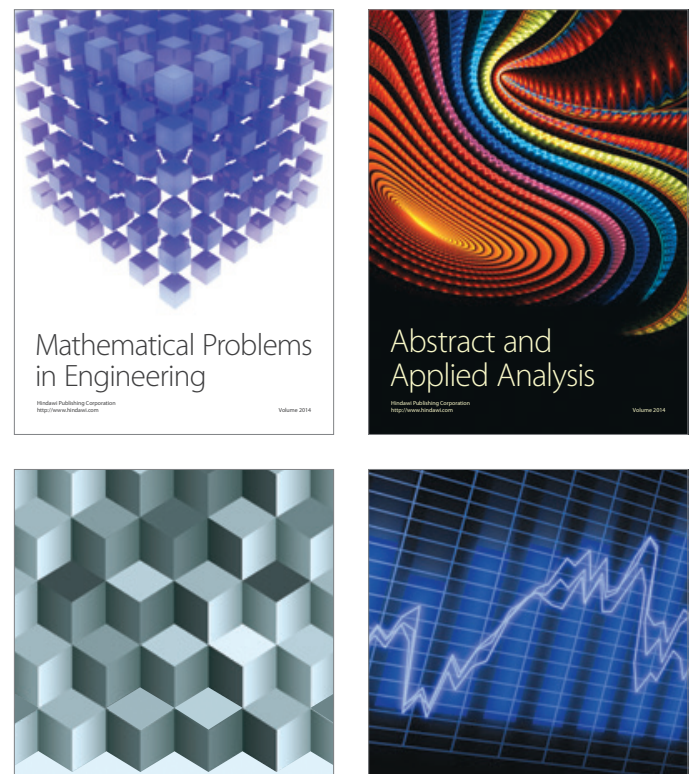

Journal of

Function Spaces

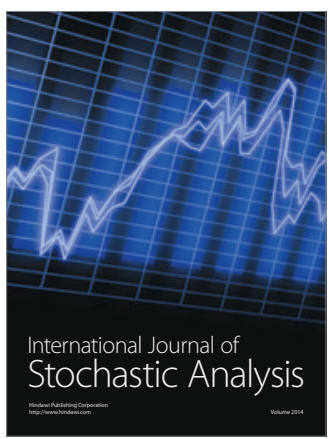

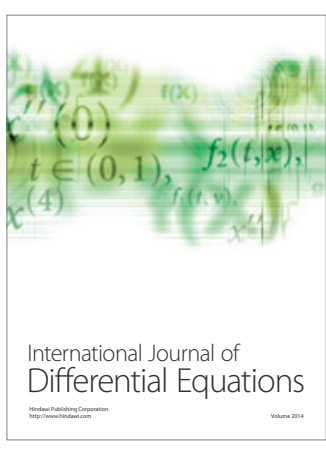
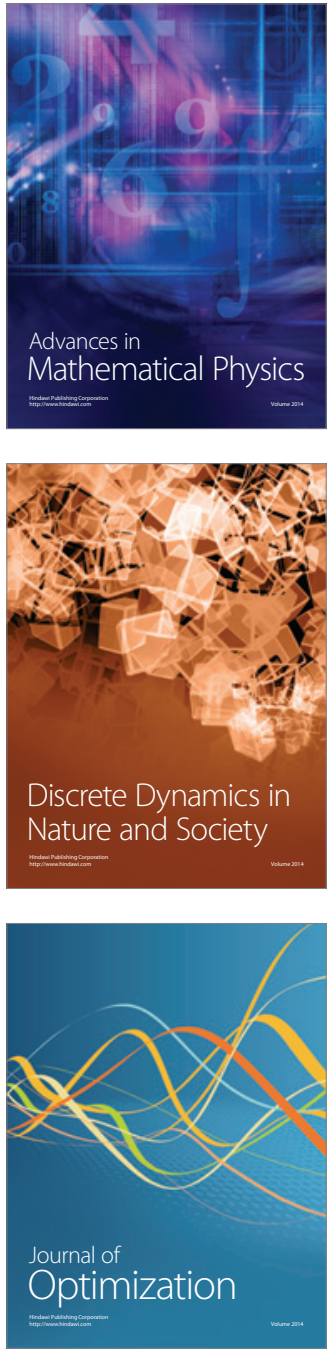